S-

\title{
Rearrangement of 4,5-Epoxy-9-trimethylsilyldecalines.
}

\author{
Application to the Synthesis of the Natural \\ Eremophilane (-)-Aristolochene
}

Gonzalo Blay, Luz Cardona, Ana M. Collado, Begoña García and José R. Pedro*

Departament de Química Orgànica, Facultat de Química, Universitat de València, 46100 Burjassot (València), Spain

\section{SUPPORTING INFORMATION}

Table of Contents:

- General experimental methods

S2

-Experimental procedures and characterization data

for compounds 3b-7b, 14-16, and 18-22

S2-S8

- ${ }^{1} \mathrm{H}$ NMR and ${ }^{13} \mathrm{C}$ NMR spectra of compounds

5a-7a, 3b-7b, 8-10, and 12-24

S9-S60 
General experimental methods. Commercial reagents and solvents were analytical grade or were purified by standard procedures, prior to use. All reactions involving air or moisture sensitive materials were carried out under argon atmosphere. Reactions were monitored by TLC analysis using Merck Silica Gel 60 F-254 thin layer plates. Flash column chromatography was performed on Merck silica gel 60, 0.040-0.063 mm. Specific optical rotations were measured in $\mathrm{CHCl}_{3}$ using sodium light (D line 589 $\mathrm{nm}$ ). IR were recorded as liquid films in $\mathrm{NaCl}$ for oils and as $\mathrm{KBr}$ discs for solids. NMR were run in $\mathrm{CDCl}_{3}$ unless otherwise stated, and referenced to the solvent as internal standard. The carbon type was determined by DEPT experiments. EIMS were run at $70 \mathrm{eV}$.

(6R,7R,9S)-(-)-9-Isopropenyl-2,6-dimethyl-7-trimethylsilylbicyclo[4.4.0]dec-1-en-3-one (3b). A solution of $\mathrm{KOH}(0.28 \mathrm{~g}, 5.0 \mathrm{mmol})$ in methanol $(8.3 \mathrm{~mL})$ was added to a solution containing $(3 R, 5 S)-$ 5-isopropenyl-2-methyl-3-trimethylsilylcyclohexanone 2 (4.5 g (20.0 mmol), EVK (3.0 mL (30.0 mmol) in methanol $(7.6 \mathrm{~mL})$. The mixture was heated at reflux temperature under argon for $16 \mathrm{~h}$. The excess $\mathrm{MeOH}$ and EVK was removed under reduced pressure. The crude was dissolved in dry benzene (13.5 mL), pyrrolidine $(0.19 \mathrm{~mL}, 2.3 \mathrm{mmol})$ added and the mixture heated under reflux with a waterseparatory Dean-Stark system for 1.5 h, under argon. After this time the mixture was acidified with $10 \%$ aqueous $\mathrm{HCl}$. The organic layer was separated, and the aqueous layer extracted with EtOAc. The combined organic layers were washed with brine and dried over $\mathrm{Na}_{2} \mathrm{SO}_{4}$. The solvent was removed under reduced pressure and chromatography (hexanes-EtOAc, 90:10) allowed to obtain $4.06 \mathrm{~g}$ (70\%) of compound 3b: oil; $[\alpha]_{\mathrm{D}}^{26}-114$ (c 1.6, $\left.\mathrm{CHCl}_{3}\right) ; \mathrm{EM} \mathrm{m/z} 290\left(\mathrm{M}^{+}, 11\right), 276$ (17), 275 (61), 235 (11), 73 (100); HRMS found 290.2061 $\left(\mathrm{M}^{+}\right), \mathrm{C}_{18} \mathrm{H}_{30} \mathrm{OSi}$ required 290.5248; IR (NaCl) 2949, 1660, 1435, 1247, 846, $835 \mathrm{~cm}^{-1}$; ${ }^{1} \mathrm{H}$ NMR (400 MHz) $\delta 4.76(1 \mathrm{H}, \mathrm{s}), 4.56(1 \mathrm{H}, \mathrm{s}), 2.85(1 \mathrm{H}, \mathrm{dt}, J=16.0,2.8 \mathrm{~Hz}), 2.52$ (1H, m), 2.45 (1H, dd, $J=14.5,5.2 \mathrm{~Hz}), 2.348 ; 2.24$ (2H, m), 1.93 (1H, ddd, $J=13.2,5.6,2.8,13.2$ Hz), 1.85 (1H, dt, $J=5.2,14.5), 1.75$ (3H, d, $J=1.2 \mathrm{~Hz}), 1.74-1.66$ (2H, m), 1.63 (3H, br s), 1.21 (3H, s), $0.91(1 \mathrm{H}, \mathrm{dd}, J=13.6,3.2 \mathrm{~Hz}), 0.02\left(9 \mathrm{H}, \mathrm{s},\left(\mathrm{CH}_{3}\right)_{3} \mathrm{Si}\right) ;{ }^{13} \mathrm{C}$ NMR $\delta 197.8(\mathrm{C}), 164.0(\mathrm{C}), 146.5(\mathrm{C})$, 
S-

3

127.7 (C), 111.1 (CH2), $40.9(\mathrm{CH}), 38.9(\mathrm{C}), 37.5\left(\mathrm{CH}_{2}\right), 33.6\left(\mathrm{CH}_{2}\right), 32.0(\mathrm{CH}), 31.3\left(\mathrm{CH}_{2}\right), 25.6$ ( $\left.\mathrm{CH}_{2}\right), 22.2\left(\mathrm{CH}_{3}\right), 21.1\left(\mathrm{CH}_{3}\right), 10.8\left(\mathrm{CH}_{3}\right), 0.3\left(3 \mathrm{CH}_{3},\left(\mathrm{CH}_{3}\right)_{3} \mathrm{Si}\right)$.

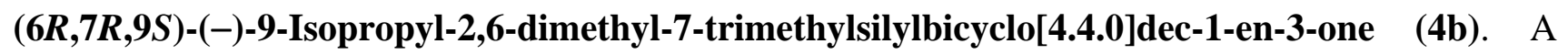
solution of compound $3 \mathbf{b}(1.20 \mathrm{~g}, 4.13 \mathrm{mmol})$ in benzene $(7.8 \mathrm{~mL})$ was added via syringe to a solution of Wilkinson's catalyst $(420 \mathrm{mg})$ in benzene $(5.5 \mathrm{~mL})$ under $\mathrm{H}_{2}$ at atmospheric pressure. The solution was stirred for $14 \mathrm{~h}$ and the solvent removed under reduced pressure. The residue was chromatographed eluting with hexane-EtOAc (9:1) to give $1.05 \mathrm{~g}$ (87\%) of compound $\mathbf{4 b}$ : white solid; mp $43-44{ }^{\circ} \mathrm{C}$; $[\alpha]_{\mathrm{D}}{ }^{25}-100$ (c 1.6, $\left.\mathrm{CHCl}_{3}\right) ; \mathrm{EM} \mathrm{m} / \mathrm{z} 292\left(\mathrm{M}^{+}, 12\right), 278$ (24), 277 (100), 249 (15), 222 (54), 73 (41); HRMS found $292.2228\left(\mathrm{M}^{+}\right), \mathrm{C}_{18} \mathrm{H}_{32} \mathrm{OSi}$ required 292.2222; IR (KBr) 1665, 1455, 1251, 859, $834 \mathrm{~cm}^{-1}$; ${ }^{1} \mathrm{H}$ NMR (400 MHz) $\delta 2.79$ (1H, br dd, $\left.J=14.8,4.4 \mathrm{~Hz}\right), 2.48$ (1H, ddd, $\left.J=17.2,15.2,5.2 \mathrm{~Hz}\right), 2.34$ (1H, ddd, $J=17.2,4.8,3.2 \mathrm{~Hz}), 2.21$ (1H, ddd, $J=14.8,4.4,1.2 \mathrm{~Hz}), 2.00$ (1H, ddd, $J=13.2,5.2,3.2$ Hz), 1.76 (3H, s), 1.80-1.45 (5H, m), 1.25 (3H, s), 0.94 (3H, d, $J=6.4$ Hz), 0.89 (1H, dd, $J=7.6,10.4$ Hz), 0.82 (3H, d, $J=6.0 \mathrm{~Hz}), 0.08\left(9 \mathrm{H}, \mathrm{s},\left(\mathrm{CH}_{3}\right)_{3} \mathrm{Si}\right) ;{ }^{13} \mathrm{C}$ NMR $\delta 198.8(\mathrm{C}), 164.3(\mathrm{C}), 128.9(\mathrm{C}), 42.6$ (CH), $39.3(\mathrm{C}), 37.9\left(\mathrm{CH}_{2}\right), 33.8\left(\mathrm{CH}_{2}\right), 33.6(\mathrm{CH}), 31.6\left(\mathrm{CH}_{2}\right), 27.1(\mathrm{CH}), 26.5\left(\mathrm{CH}_{2}\right), 22.0\left(\mathrm{CH}_{3}\right), 21.1$ $\left(\mathrm{CH}_{3}\right), 20.3\left(\mathrm{CH}_{3}\right), 11.3\left(\mathrm{CH}_{3}\right), 0.5\left(3 \mathrm{CH}_{3},\left(\mathrm{CH}_{3}\right)_{3} \mathrm{Si}\right)$.

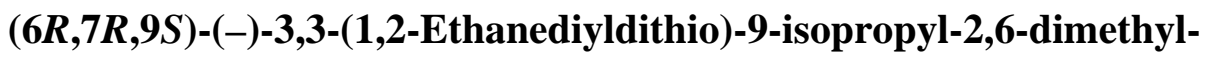

trimethylsilylbicyclo[4.4.0]dec-1-ene (5b). By the same procedure described for the synthesis of 5a, after 15 h, from $4 \mathbf{b}$ (1.05 g, 3.59 mmol) were obtained 1.21 g (92\%) of thioketal 5b: white solid; mp 68-70 ${ }^{\circ} \mathrm{C}$ (hexane-EtOAc); $[\alpha]_{\mathrm{D}}{ }^{25}-116$ (c 1.5, $\left.\mathrm{CHCl}_{3}\right) ; \mathrm{EM} \mathrm{m/z} 368\left(\mathrm{M}^{+}, 86\right), 325$ (17), 308 (51), 307 (94), 293 (22), 211 (16), 133 (18), 73 (100); HRMS found $368.023\left(\mathrm{M}^{+}\right), \mathrm{C}_{20} \mathrm{H}_{36} \mathrm{~S}_{2}$ Si required 368.2028; IR (KBr) 1448, 1257, $844 \mathrm{~cm}^{-1} ;{ }^{1} \mathrm{H}$ NMR (400 MHz) $\delta 3.45-3.10$ (4H, m, $\left.\mathrm{SCH}_{2} \mathrm{CH}_{2} \mathrm{~S}\right), 2.60(1 \mathrm{H}$, br d, $J$ = 14.3 Hz), 2.30-2.10 (2H, m), 2.01 (1H, ddd, $J=14.4,4.8,1.2 \mathrm{~Hz}), 1.89(3 \mathrm{H}, \mathrm{d}, J=1.2 \mathrm{~Hz}), 1.80(1 \mathrm{H}$, ddd, $J=3.0,4.8,13.4 \mathrm{~Hz}), 1.70-1.20(3 \mathrm{H}, \mathrm{m}), 1.12(3 \mathrm{H}, \mathrm{s}), 0.94(3 \mathrm{H}, \mathrm{d}, J=6.4 \mathrm{~Hz}), 0.79$ (3H, d, $J=$ 6.4 Hz), $0.74(1 \mathrm{H}$, dd, $J=10.4,6.8 \mathrm{~Hz}), 0.04\left(9 \mathrm{H}, \mathrm{s},\left(\mathrm{CH}_{3}\right)_{3} \mathrm{Si}\right) ;{ }^{13} \mathrm{C}$ NMR $\delta 142.3(\mathrm{C}), 125.6(\mathrm{C}), 72.6$ 
S-

(C), $43.2(\mathrm{CH}), 41.1\left(\mathrm{CH}_{2}\right), 39.8\left(2 \mathrm{CH}_{2}, \mathrm{SCH}_{2} \mathrm{CH}_{2} \mathrm{~S}\right), 39.6\left(\mathrm{CH}_{2}\right), 38.1(\mathrm{C}), 34.1(\mathrm{CH}), 30.1\left(\mathrm{CH}_{2}\right), 26.5$

$\left(\mathrm{CH}_{2}\right), 25.9(\mathrm{CH}), 22.9\left(\mathrm{CH}_{3}\right), 22.7\left(\mathrm{CH}_{3}\right), 20.5\left(\mathrm{CH}_{3}\right), 17.0\left(\mathrm{CH}_{3}\right), 0.5\left(3 \mathrm{CH}_{3},\left(\mathrm{CH}_{3}\right)_{3} \mathrm{Si}\right)$.

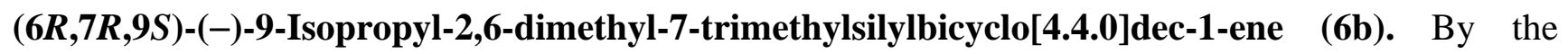
same procedure described for the synthesis of $\mathbf{6 a}$, from $5 \mathbf{b}(1.20 \mathrm{~g}, 3.25 \mathrm{mmol})$ were obtained $703 \mathrm{mg}$ (78\%) of alkene 6b: oil; $[\alpha]_{\mathrm{D}}^{22}-83$ (c 1.5, $\mathrm{CHCl}_{3}$ ); EM m/z 278 (M+33), 264 (20), 263 (76), 235 (16), 190 (17), 189 (100), 177 (20), 123 (32), 122 (37), 73 (82); HRMS found $278.2432\left(\mathrm{M}^{+}\right.$), $\mathrm{C}_{18} \mathrm{H}_{34} \mathrm{Si}$ required 278.2430; IR (NaCl) 1450, 1381, 1255, $844 \mathrm{~cm}^{-1} ;{ }^{1} \mathrm{H}$ NMR (400 MHz) $\delta 2.61(1 \mathrm{H}, \mathrm{br} \mathrm{d}, J=$ $14.0 \mathrm{~Hz}), 1.60(3 \mathrm{H}, \mathrm{s}), 1.54(3 \mathrm{H}, \mathrm{m}), 1.10(3 \mathrm{H}, \mathrm{s}), 0.92(3 \mathrm{H}, \mathrm{d}, J=6.4 \mathrm{~Hz}), 0.79(3 \mathrm{H}, \mathrm{d}, J=6.8 \mathrm{~Hz})$, $0.73(1 \mathrm{H}, \mathrm{t}, J=8.4 \mathrm{~Hz}), 0.03\left(9 \mathrm{H}, \mathrm{s},\left(\mathrm{CH}_{3}\right)_{3} \mathrm{Si}\right) ;{ }^{13} \mathrm{C}$ NMR $\delta 135.2(\mathrm{C}), 124.4(\mathrm{C}), 43.2(\mathrm{CH}), 40.7$ ( $\left.\mathrm{CH}_{2}\right), 38.2(\mathrm{C}), 34.0(\mathrm{CH}), 32.9\left(\mathrm{CH}_{2}\right), 28.7\left(\mathrm{CH}_{2}\right), 27.0\left(\mathrm{CH}_{2}\right), 25.7(\mathrm{CH}), 23.3\left(\mathrm{CH}_{3}\right), 22.3\left(\mathrm{CH}_{3}\right)$, $20.5\left(\mathrm{CH}_{3}\right), 20.1\left(\mathrm{CH}_{3}\right), 19.1\left(\mathrm{CH}_{2}\right), 0.6\left(3 \mathrm{CH}_{3},\left(\mathrm{CH}_{3}\right)_{3} \mathrm{Si}\right)$.

(1S,2R,6R,7R,9S)-(-)-1,2-Epoxy-9-isopropyl-2,6-dimethyl-7-(trimethylsilyl)bicyclo[4.4.0]decane (7b). By the same procedure described for the synthesis of 7a, from $\mathbf{6 b}$ (114 mg, $0.41 \mathrm{mmol})$ were obtained $101 \mathrm{mg}$ (83\%) of epoxide 7b: oil; $[\alpha]_{\mathrm{D}}{ }^{18}-19$ (c 1.9, $\left.\mathrm{CHCl}_{3}\right)$; EM m/z $294\left(\mathrm{M}^{+}, 13\right), 251$ (26), 159 (18), 143 (51), 119 (16), 105 (30), 73 (100); HRMS: 294.2376 (M+), $\mathrm{C}_{18} \mathrm{H}_{34} \mathrm{OSi}$ required 294.2379; IR (NaCl) 1453, 1375, 1248, 861, $832 \mathrm{~cm}^{-1} ;{ }^{1} \mathrm{H}$ NMR (300 MHz) $\delta 1.82(1 \mathrm{H}, \mathrm{dt}, J=17.5,7.0 \mathrm{~Hz}), 1.24$ (3H, s), 1.03 (3H, s), 0.97 (1H, dd, $J=15.4,5.7 \mathrm{~Hz}), 0.87(3 \mathrm{H}, \mathrm{d}, J=7.0 \mathrm{~Hz}), 0.82$ (3H, d, $J=7.0 \mathrm{~Hz})$, $0.01\left(9 \mathrm{H}, \mathrm{s},\left(\mathrm{CH}_{3}\right)_{3} \mathrm{Si}\right) ;{ }^{13} \mathrm{C} \mathrm{NMR} \delta 68.3(\mathrm{C}), 65.0(\mathrm{C}), 39.9(\mathrm{CH}), 37.6\left(\mathrm{CH}_{2}\right), 37.1(\mathrm{C}), 32.0\left(\mathrm{CH}_{2}\right)$, 30.6 ( $\left(\mathrm{CH}_{2}\right), 29.9(\mathrm{CH}), 28.7(\mathrm{CH}), 25.9\left(\mathrm{CH}_{2}\right), 21.8\left(\mathrm{CH}_{3}\right), 21.7\left(\mathrm{CH}_{3}\right), 20.8\left(\mathrm{CH}_{3}\right), 20.1\left(\mathrm{CH}_{3}\right), 16.8$ $\left(\mathrm{CH}_{2}\right), 0.5\left(3 \mathrm{CH}_{3},\left(\mathrm{CH}_{3}\right)_{3} \mathrm{Si}\right)$.

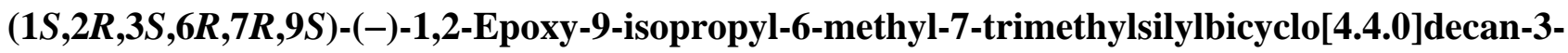

ol (14). By the same procedure described for the synthesis of 7a, from 8 (60 mg, $0.23 \mathrm{mmol})$ were obtained 47 mg g (69\%) of compound 14: oil; $[\alpha]_{\mathrm{D}}^{29}-12$ (c 0.6, $\left.\mathrm{CHCl}_{3}\right) ; \mathrm{EM} \mathrm{m/z} 296\left(\mathrm{M}^{+}, 2\right), 226$ (100), 145 (63), 119 (24), 73 (92); HRMS found $296.2176\left(\mathrm{M}^{+}\right), \mathrm{C}_{17} \mathrm{H}_{32} \mathrm{O}_{2} \mathrm{Si}$ required 296.2172; IR (NaCl) 3428, 1249, 861, $831 \mathrm{~cm}^{-1} ;{ }^{1} \mathrm{H}$ NMR (400 MHz) $\delta 3.94$ (1H, dd, $\left.J=8.5,6.7 \mathrm{~Hz}\right), 2.87$ (1H, s), 
S-

$2.06(1 \mathrm{H}, \mathrm{dd}, J=13.6,4.8 \mathrm{~Hz}), 1.9-1.7(3 \mathrm{H}, \mathrm{m}), 1.85(1 \mathrm{H}, \mathrm{m}), 1.63(1 \mathrm{H}, \mathrm{td}, J=14.0,4.4 \mathrm{~Hz}), 1.5-1.3$

(3H, m), 1.3-1.1 (1H, m), 1.25 (1H, d, $J=13.6 \mathrm{~Hz}$ ), 1.06 (3H, s), 0.90 (3H, d, $J=6.8 \mathrm{~Hz}$ ), 0.84 (3H, d, $J$

= $6.8 \mathrm{~Hz}), 0.04\left(9 \mathrm{H}, \mathrm{s},\left(\mathrm{CH}_{3}\right)_{3} \mathrm{Si}\right) ;{ }^{13} \mathrm{C} \mathrm{NMR} \delta 66.8(\mathrm{C}), 66.5(\mathrm{CH}), 64.4(\mathrm{CH}), 43.0(\mathrm{CH}), 36.5(\mathrm{C}), 33.5$

$\left(\mathrm{CH}_{2}\right)$, $29.6\left(\mathrm{CH}_{2}\right), 26.5(\mathrm{CH}), 26.3(\mathrm{CH}), 26.2\left(\mathrm{CH}_{2}\right), 26.1\left(\mathrm{CH}_{2}\right), 21.6\left(\mathrm{CH}_{3}\right), 21.2\left(\mathrm{CH}_{3}\right), 20.8\left(\mathrm{CH}_{3}\right)$, $0.4\left(3 \mathrm{CH}_{3},\left(\mathrm{CH}_{3}\right)_{3} \mathrm{Si}\right)$.

Rearrangement of compound 7a: (1S,5R,9R)-(-)-9-isopropyl-1-methylbicyclo[4.4.0]dec-6-en-5-ol (15) and (1R,2R,9R)-(-)-9-isopropyl-1-methylbicyclo[4.4.0]dec-6-en-2-ol (16). A solution of $\mathrm{BF}_{3} \cdot \mathrm{Et}_{2} \mathrm{O}(46 \mu \mathrm{L}, 0.36 \mathrm{mmol})$ in dry $\mathrm{CH}_{3} \mathrm{CN}(4.6 \mathrm{~mL})$ was added dropwise to a solution of epoxide $7 \mathbf{a}$ (81 mg, $0.29 \mathrm{mmol}$ ) in dry $\mathrm{CH}_{3} \mathrm{CN}(4.6 \mathrm{~mL})$ cooled at $-25{ }^{\circ} \mathrm{C}$ under argon. After $15 \mathrm{~min}$, the reaction was quenched with saturated aqueous $\mathrm{NaHCO}_{3}$ and extracted with EtOAc, washed with brine and dried. Removal of the solvent under reduced pressure gave $45 \mathrm{mg}$ (75\%) of a ca. 8:2 mixture of compounds 15 and 16 which were separated by HPLC (normal phase, hexanes-EtOAc, 95:5).

Major component 15: oil; $[\alpha]_{\mathrm{D}}{ }^{25}-12$ (c 0.6, $\left.\mathrm{CHCl}_{3}\right)$; EM m/z 208 (M+15), 190 (18), 165 (20), 147 (100), 121 (22), 109 (20), 91 (19); HRMS found $208.1818\left(\mathrm{M}^{+}\right), \mathrm{C}_{14} \mathrm{H}_{24} \mathrm{O}$ required 208.1827; IR ( $\left.\mathrm{NaCl}\right)$ 3401, 1457, $1075 \mathrm{~cm}^{-1}$; ${ }^{1} \mathrm{H}$ NMR (400 MHz) $\delta 5.36$ (1H, m, $J=2.2 \mathrm{~Hz}$ ), 4.03 (1H, t, $J=7.2 \mathrm{~Hz}$ ), 2.37$2.30(2 \mathrm{H}, \mathrm{m}), 2.01-1.86(4 \mathrm{H}, \mathrm{m}), 1.72-1.66$ (2H, m), 1.60-1.45 (5H, m), 1.01 (3H, s), 0.86 (3H, d, $J=$ 6.6 Hz), 0.85 (3H, d, $J=6.6 \mathrm{~Hz}) ;{ }^{13} \mathrm{C}$ NMR $\delta 149.7(\mathrm{C}), 122.5(\mathrm{CH}), 77.7(\mathrm{CH}), 47.3(\mathrm{C}), 46.4(\mathrm{CH})$, $36.8\left(\mathrm{CH}_{2}\right), 36.4\left(\mathrm{CH}_{2}\right), 35.0\left(\mathrm{CH}_{2}\right), 33.6(\mathrm{CH}), 31.4\left(\mathrm{CH}_{2}\right), 21.2\left(\mathrm{CH}_{3}\right), 21.0\left(\mathrm{CH}_{3}\right), 19.2\left(\mathrm{CH}_{2}\right), 18.1$ $\left(\mathrm{CH}_{3}\right)$.

Minor component 16: oil; $[\alpha]_{\mathrm{D}}^{25}-82$ (c 0.7, $\left.\mathrm{CHCl}_{3}\right)$; EM m/z 208 (M+1 11), 190 (16), 147 (100), 105 (25), 95 (21); HRMS found $208.1821\left(\mathrm{M}^{+}\right), \mathrm{C}_{14} \mathrm{H}_{24} \mathrm{O}$ required 208.1827; IR ( $\left.\mathrm{NaCl}\right) 3382,1464,1054$, $1009 \mathrm{~cm}^{-1} ;{ }^{1} \mathrm{H}$ NMR (300 MHz) $\delta 5.40(1 \mathrm{H}, \mathrm{m}, J=2.0 \mathrm{~Hz}), 3.26(1 \mathrm{H}, \mathrm{dd}, J=11.5,4.2 \mathrm{~Hz}), 2.05(1 \mathrm{H}$, m), 2.01-1.90 (2H, m), $1.86(1 \mathrm{H}, \mathrm{td}, J=12.5,1.9 \mathrm{~Hz}), 1.76-1.67(3 \mathrm{H}, \mathrm{m}), 1.02(3 \mathrm{H}, \mathrm{s}), 0.90$ (3H, d, $J=$ 6.4 Hz), 0.86 (3H, d, $J=6.6 \mathrm{~Hz}) ;{ }^{13} \mathrm{C}$ NMR $\delta 141.5(\mathrm{C}), 121.8(\mathrm{CH}), 80.4(\mathrm{CH}), 41.3(\mathrm{C}), 40.2\left(\mathrm{CH}_{2}\right)$, 
S-

6

36.1 (CH), $32.4(\mathrm{CH}), 31.3\left(\mathrm{CH}_{2}\right), 30.6\left(\mathrm{CH}_{2}\right), 29.4\left(\mathrm{CH}_{2}\right), 24.8\left(\mathrm{CH}_{2}\right), 20.0\left(\mathrm{CH}_{3}\right), 19.4\left(\mathrm{CH}_{3}\right), 17.1$

$\left(\mathrm{CH}_{3}\right)$.

\section{Rearrangement of compound 7b: $(1 R, 2 R, 9 R)-(-)-9$-isopropyl-1,2-dimethylbicyclo[4.4.0]dec-6-}

en-2-ol (18). The same procedure as described for the rearrangement of 7a was employed. From compound 7b (15 mg, 0.05 mmol), after column chromatography (hexanes-EtOAc, 98:2) were obtained $5.2 \mathrm{mg}$ (58\%) of compound 18: oil; $[\alpha]_{\mathrm{D}}^{25}-49$ (c 0.6, $\left.\mathrm{CHCl}_{3}\right) ; \mathrm{EM} \mathrm{m/z} 222\left(\mathrm{M}^{+}, 59\right), 204$ (32), 164 (100), 161 (89), HRMS found $222.1979\left(\mathrm{M}^{+}\right), \mathrm{C}_{15} \mathrm{H}_{26} \mathrm{O}$ required 222.1984; IR (NaCl) 3430, 1452, 1369, 1095, 928, $809 \mathrm{~cm}^{-1}$; ${ }^{1} \mathrm{H}$ NMR (400 MHz) $\delta 5.37$ (1H, dt, $\left.J=7.2,2.2 \mathrm{~Hz}\right), 2.20(1 \mathrm{H}, \mathrm{m}), 2.0-1.8$ (3H, m), 1.7-1.3 (8H, m), 1.19 (3H, s), $1.16(3 \mathrm{H}, \mathrm{s}), 0.89$ (3H, d, $J=6.5 \mathrm{~Hz}), 0.87(3 \mathrm{H}, \mathrm{d}, J=7.0 \mathrm{~Hz}) ;{ }^{13} \mathrm{C}$ NMR $\delta 141.6(\mathrm{C}), 121.7(\mathrm{C}), 75.9(\mathrm{C}), 44.4(\mathrm{C}), 37.5\left(\mathrm{CH}_{2}\right), 36.8(\mathrm{CH}), 34.1\left(\mathrm{CH}_{2}\right), 32.5(\mathrm{CH}), 30.8$ (CH$), 29.5\left(\mathrm{CH}_{2}\right), 24.9\left(\mathrm{CH}_{3}\right), 23.3\left(\mathrm{CH}_{2}\right), 20.7\left(\mathrm{CH}_{3}\right), 20.1\left(\mathrm{CH}_{3}\right), 19.6\left(\mathrm{CH}_{3}\right)$.

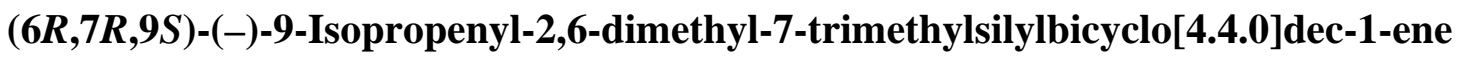

(19). A suspension of $\mathrm{LiAlH}_{4}(906 \mathrm{mg}, 23.9 \mathrm{mmol})$ in dry diethyl ether (19 mL) was treated with a solution of $\mathrm{AlCl}_{3}(9.6 \mathrm{~g}, 71.6 \mathrm{mmol})$ in dry diethyl ether $(44 \mathrm{~mL})$ at $0{ }^{\circ} \mathrm{C}$ under argon. After 5 min compound $3 \mathbf{b}$ (1.63 g, $5.60 \mathrm{mmol})$ dissolved in dry diethyl ether $(19.4 \mathrm{~mL})$ was added and the mixture stirred at $0{ }^{\circ} \mathrm{C}$ for $1.5 \mathrm{~h}$. After this time, the reaction was quenched with ice and extracted with diethyl ether. The organic layer was washed with brine and dried. Column chromatography (pentane) gave 835 mg (54\%) of compound 19: oil, $[\alpha]_{\mathrm{D}}^{18}-79$ (c 1.3, $\left.\mathrm{CHCl}_{3}\right)$; EM m/z 276 (M+1, 45), 262 (11), 261 (40), 187 (30), 175 (32), 147 (20), 123 (17), 73 (100); HRMS found $276.2282\left(\mathrm{M}^{+}\right), \mathrm{C}_{18} \mathrm{H}_{32} \mathrm{Si}$ required 276.5363; IR (NaCl) 3732, 2939, 2346, 1619, 1445, 1251, $845 \mathrm{~cm}^{-1}$; ${ }^{1} \mathrm{H}$ NMR (400 MHz) $\delta 4.79$ (1H, s), 4.75 (1H, br s), 2.74 (1H, dt, $J=14.8,2.4 \mathrm{~Hz}), 2.39$ (1H, br s), 2.1-1.4 (5H, m), $1.85(1 \mathrm{H}, \mathrm{td}, J=13.6,5.2 \mathrm{~Hz}), 1.68$ (3H, s), 1.61 (3H, s), $1.29(1 \mathrm{H}, \mathrm{dt}, J=3.2,12.8 \mathrm{~Hz}), 1.10(3 \mathrm{H}, \mathrm{s}), 0.93(1 \mathrm{H}, \mathrm{m}), 0.87$ (1H, dd, $J=3.2$, 13.6 Hz), $0.02\left(9 \mathrm{H}, \mathrm{s},\left(\mathrm{CH}_{3}\right)_{3} \mathrm{Si}\right) ;{ }^{13} \mathrm{C} \mathrm{NMR} \delta 147.8(\mathrm{C}), 135.0(\mathrm{C}), 123.1(\mathrm{C}), 110.8\left(\mathrm{CH}_{2}\right), 41.5(\mathrm{CH})$, $40.2\left(\mathrm{CH}_{2}\right), 37.9(\mathrm{C}), 33.2(\mathrm{CH}), 32.6\left(\mathrm{CH}_{2}\right), 28.7\left(\mathrm{CH}_{2}\right), 26.5\left(\mathrm{CH}_{2}\right), 23.2\left(\mathrm{CH}_{3}\right), 23.0\left(\mathrm{CH}_{3}\right), 19.9$ $\left(\mathrm{CH}_{3}\right), 19.0\left(\mathrm{CH}_{2}\right), 0.7\left(3 \mathrm{CH}_{3},\left(\mathrm{CH}_{3}\right)_{3} \mathrm{Si}\right)$. 
S-

(1S,2R,6R,7R,9S)-(-)-1,2-Epoxy-9-isopropenyl-2,6-dimethyl-7-trimethylsilylbicyclo[4.4.0]decane

(20). By the same procedure described for the synthesis of $\mathbf{7 a}$, after $1 \mathrm{~h}$ at $0{ }^{\circ} \mathrm{C}$, from $\mathbf{1 9}(1.21 \mathrm{~g}, 4.37$ mmol) were obtained after column chromatography eluting with hexanes-EtOAc (from 100:0 to 97:3) $890 \mathrm{mg}$ (70\%) of monoepoxide 20 and $61 \mathrm{mg}$ (5\%) of diepoxide 21.

20: oil; $[\alpha]_{\mathrm{D}}{ }^{27}-63$ (c 1.7, $\left.\mathrm{CHCl}_{3}\right) ; \mathrm{EM} \mathrm{m/z} 292$ (M+ 38), 219 (13), 218 (15), 187 (21), 161 (28), 160 (38), 159 (23), 145 (35), 143 (100), 133 (33), 73 (71); HRMS found $292.2214\left(\mathrm{M}^{+}\right.$), $\mathrm{C}_{18} \mathrm{H}_{32} \mathrm{OSi}$ required 292.3308; IR (NaCl) 2945, 1454, 1374, 1248, $831 \mathrm{~cm}^{-1} ;{ }^{1} \mathrm{H}$ NMR (400 MHz) $\delta 4.71$ (1H, s), 4.69 (1H, s), $2.55(1 \mathrm{H}, \mathrm{m}), 1.95-1.75(3 \mathrm{H}, \mathrm{m}), 1.69(3 \mathrm{H}, \mathrm{s}), 1.65-1.00(7 \mathrm{H}, \mathrm{m}), 1.23(3 \mathrm{H}, \mathrm{s}), 1.11(1 \mathrm{H}, \mathrm{dd}, J=$ 13.6, $5.6 \mathrm{~Hz}), 1.06$ (3H, s), $0.03\left(9 \mathrm{H}, \mathrm{s},\left(\mathrm{CH}_{3}\right)_{3} \mathrm{Si}\right) ;{ }^{13} \mathrm{C} \mathrm{NMR} \delta 149.5(\mathrm{C}), 109.0(\mathrm{CH} 2), 68.0(\mathrm{C}) 63.9$ (C), $38.3\left(\mathrm{CH}_{2}\right), 38.0(\mathrm{CH}), 36.5(\mathrm{C}), 32.1\left(\mathrm{CH}_{2}\right), 31.8\left(\mathrm{CH}_{2}\right), 31.0(\mathrm{CH}), 27.0\left(\mathrm{CH}_{2}\right), 21.5\left(\mathrm{CH}_{3}\right), 20.9$ $\left(\mathrm{CH}_{3}\right), 20.2\left(\mathrm{CH}_{3}\right), 16.5\left(\mathrm{CH}_{2}\right), 0.5\left(3 \mathrm{CH}_{3},\left(\mathrm{CH}_{3}\right)_{3} \mathrm{Si}\right)$.

21: oil; $[\alpha]_{\mathrm{D}}{ }^{27}-51$ (c 0.9, $\left.\mathrm{CHCl}_{3}\right) ; \mathrm{EM} \mathrm{m/z} 308\left(\mathrm{M}^{+}, 12\right), 160$ (16), 159 (15), 156 (18), 145 (21), 143 (53), 119 (20), 118 (20), 105 (18), 73 (100); HRMS found $308.2169\left(\mathrm{M}^{+}\right), \mathrm{C}_{18} \mathrm{H}_{32} \mathrm{O}_{2} \mathrm{Si}$ required 308.3257; IR (NaCl) 2945, 1453, 1375, 1248, $832 \mathrm{~cm}^{-1} ;{ }^{1} \mathrm{H}$ NMR (400 MHz) $\delta 2.65(1 \mathrm{H}, \mathrm{d}, J=4.7$ Hz), $2.48(1 \mathrm{H}, \mathrm{d}, J=4.7 \mathrm{~Hz}), 2.03(1 \mathrm{H}, \mathrm{m}), 1.88$ (1H, td, $J=4.4,14.4 \mathrm{~Hz}), 1.26(3 \mathrm{H}, \mathrm{s}), 1.25$ (3H, s) $1.01(3 \mathrm{H}, \mathrm{s}), 0.02\left(9 \mathrm{H}, \mathrm{s},\left(\mathrm{CH}_{3}\right)_{3} \mathrm{Si}\right) ;{ }^{13} \mathrm{C} \mathrm{NMR} \delta 67.7(\mathrm{C}), 63.6(\mathrm{C}), 59.6(\mathrm{C}), 52.3(\mathrm{CH} 2), 39.1\left(\mathrm{CH}_{2}\right)$, $36.4(\mathrm{C}), 35.5(\mathrm{CH}), 32.4\left(\mathrm{CH}_{2}\right), 31.2(\mathrm{CH}), 30.1\left(\mathrm{CH}_{2}\right), 23.9\left(\mathrm{CH}_{2}\right), 20.6\left(\mathrm{CH}_{3}\right), 19.9\left(\mathrm{CH}_{3}\right), 19.5$ $\left(\mathrm{CH}_{3}\right), 16.6\left(\mathrm{CH}_{2}\right), 0.5\left(3 \mathrm{CH}_{3},\left(\mathrm{CH}_{3}\right)_{3} \mathrm{Si}\right)$.

(1R,2R,9R)-(-)-9-Isopropenyl-1,2-dimethylbicyclo[4.4.0]dec-6-en-2-ol (22). A solution of $\mathrm{TiF}_{4}$ (220 mg, $1.79 \mathrm{mmol}$ ) in dry acetonitrile (20 mL) was added dropwise to a solution of epoxide 20 (360 $\mathrm{mg}, 1.23 \mathrm{mmol})$ in dry acetonitrile $(20 \mathrm{~mL})$ at $-20{ }^{\circ} \mathrm{C}$ under argon. After $30 \mathrm{~min}$, the reaction was diluted with $\mathrm{CH}_{2} \mathrm{Cl}_{2}$, washed with cold saturated aqueous $\mathrm{NaHCO}_{3}$ and brine, and dried. Evaporation of the solvent and column chromatography eluting with hexanes-diethyl ether (from 100:0 to 70:30) gave 97.4 mg (36\%) of 22: oil; $[\alpha]_{\mathrm{D}}{ }^{28}-53$ (c 1.1, $\left.\mathrm{CHCl}_{3}\right)$; EM m/z 220 (M+ ${ }^{+}$54), 202 (25), 187 (16), 177 (18), 162 (100), 160 (23), 159 (89), 135 (60), 119 (79); HRMS found $220.1819\left(\mathrm{M}^{+}\right.$), $\mathrm{C}_{15} \mathrm{H}_{24} \mathrm{O}$ required 
8 -

220.3540; IR (NaCl) 3456, 2935, 2349, 1654, 1450, 1367, 115, $878 \mathrm{~cm}^{-1} ;{ }^{1} \mathrm{H}$ NMR $\left(\mathrm{CDCl}_{3}, 400 \mathrm{MHz}\right)$

$\delta 5.39(1 \mathrm{H}, \mathrm{dt}, J=6.3,2.1 \mathrm{~Hz}), 4.70(2 \mathrm{H}, \mathrm{br} \mathrm{s}), 2.30-1.75(5 \mathrm{H}, \mathrm{m}), 1.72(3 \mathrm{H}, \mathrm{s}), 1.70-1.20(7 \mathrm{H}, \mathrm{m}), 1.20$

(3H, s), 1.19 (3H, s); ${ }^{1} \mathrm{H}$ NMR (DMSO-d 6 , $\left.300 \mathrm{MHz}\right) \delta$ 4.33, (1H, dt, $J=6.3,2.1 \mathrm{~Hz}$ ), 4.68 (2H, s),

$4.13(1 \mathrm{H}, \mathrm{s}, \mathrm{OH}), 2.2-1.7$ (5H, m), 1.69 (3H, s), 1.6-1.1 (6H, m), 1.08 (3H, s), 1.05 (3H, s); ${ }^{13} \mathrm{C}$ NMR

$\left(\mathrm{CDCl}_{3}\right) \delta 150.2(\mathrm{C}), 141.6(\mathrm{C}), 121.3(\mathrm{CH}), 108.4\left(\mathrm{CH}_{2}\right), 75.8(\mathrm{C}), 44.7(\mathrm{C}), 37.9(\mathrm{CH}), 37.4\left(\mathrm{CH}_{2}\right)$, 35.7 ( $\left(\mathrm{CH}_{2}\right)$, $31.2\left(\mathrm{CH}_{2}\right), 30.8\left(\mathrm{CH}_{2}\right), 24.9\left(\mathrm{CH}_{3}\right) 23.3\left(\mathrm{CH}_{2}\right), 21.1\left(\mathrm{CH}_{3}\right), 20.8\left(\mathrm{CH}_{3}\right)$. 


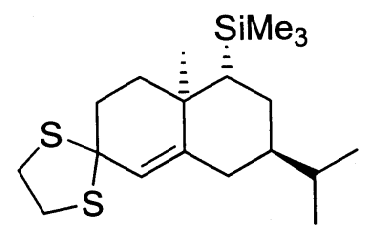

Compound 5a

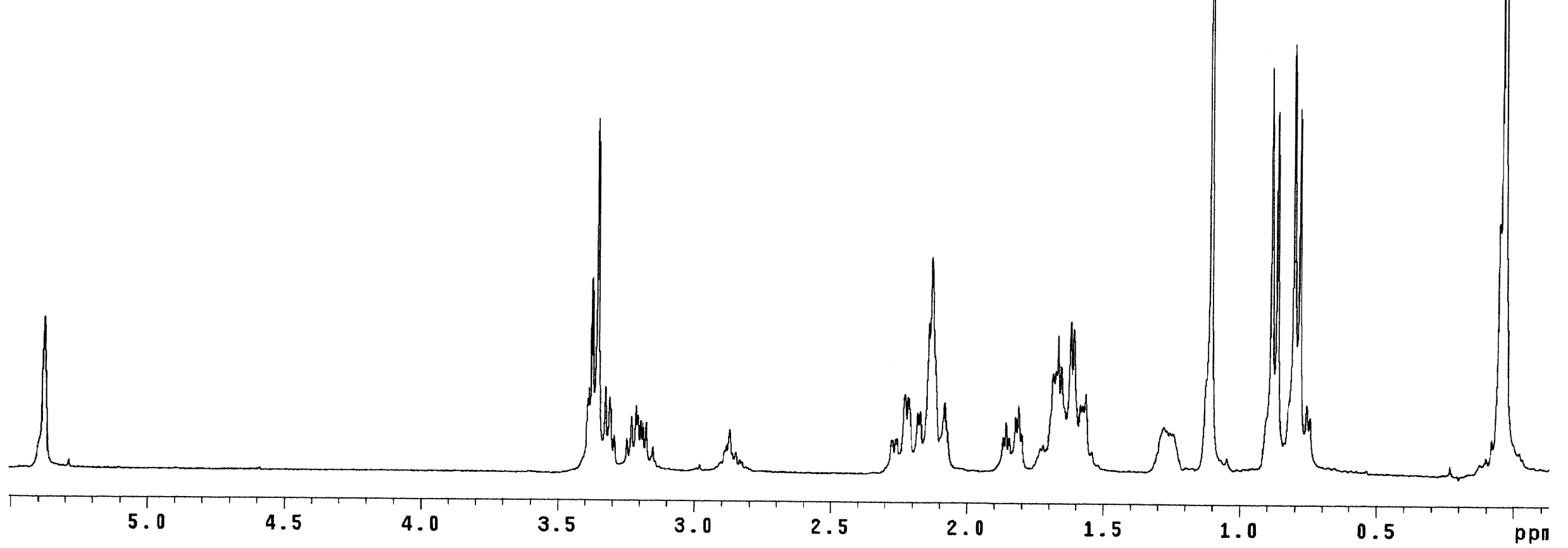




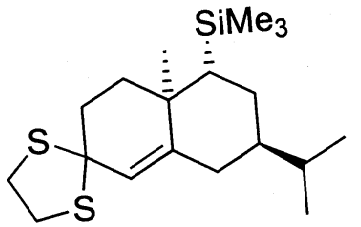

Compound 5a

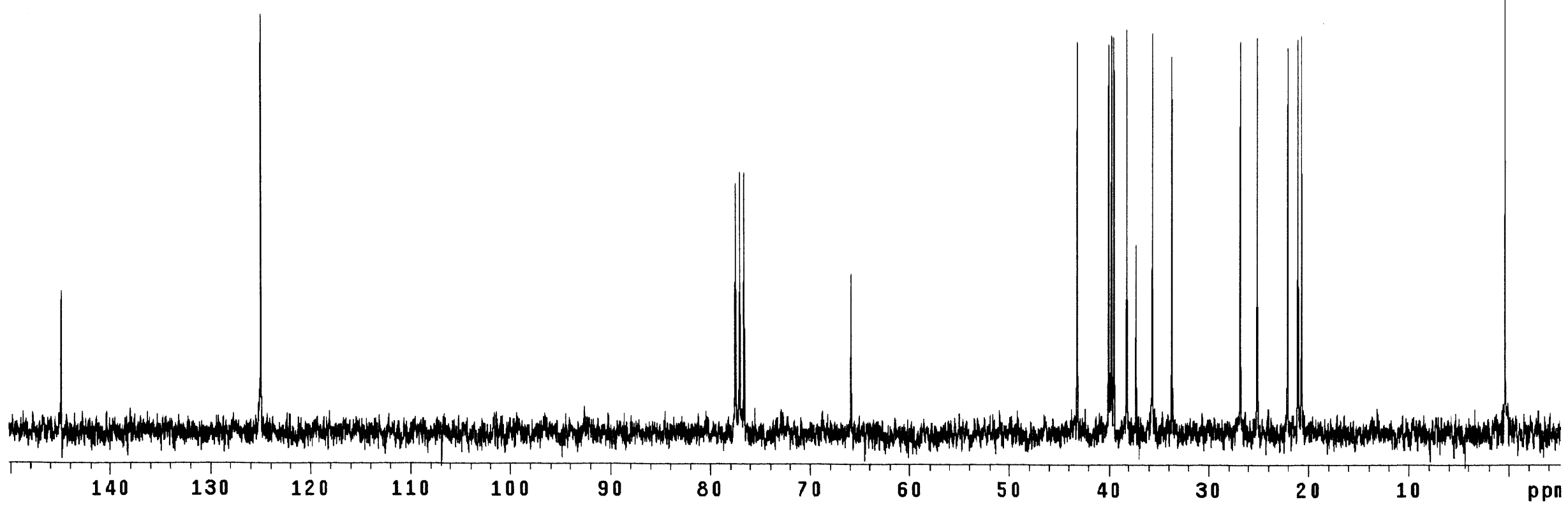




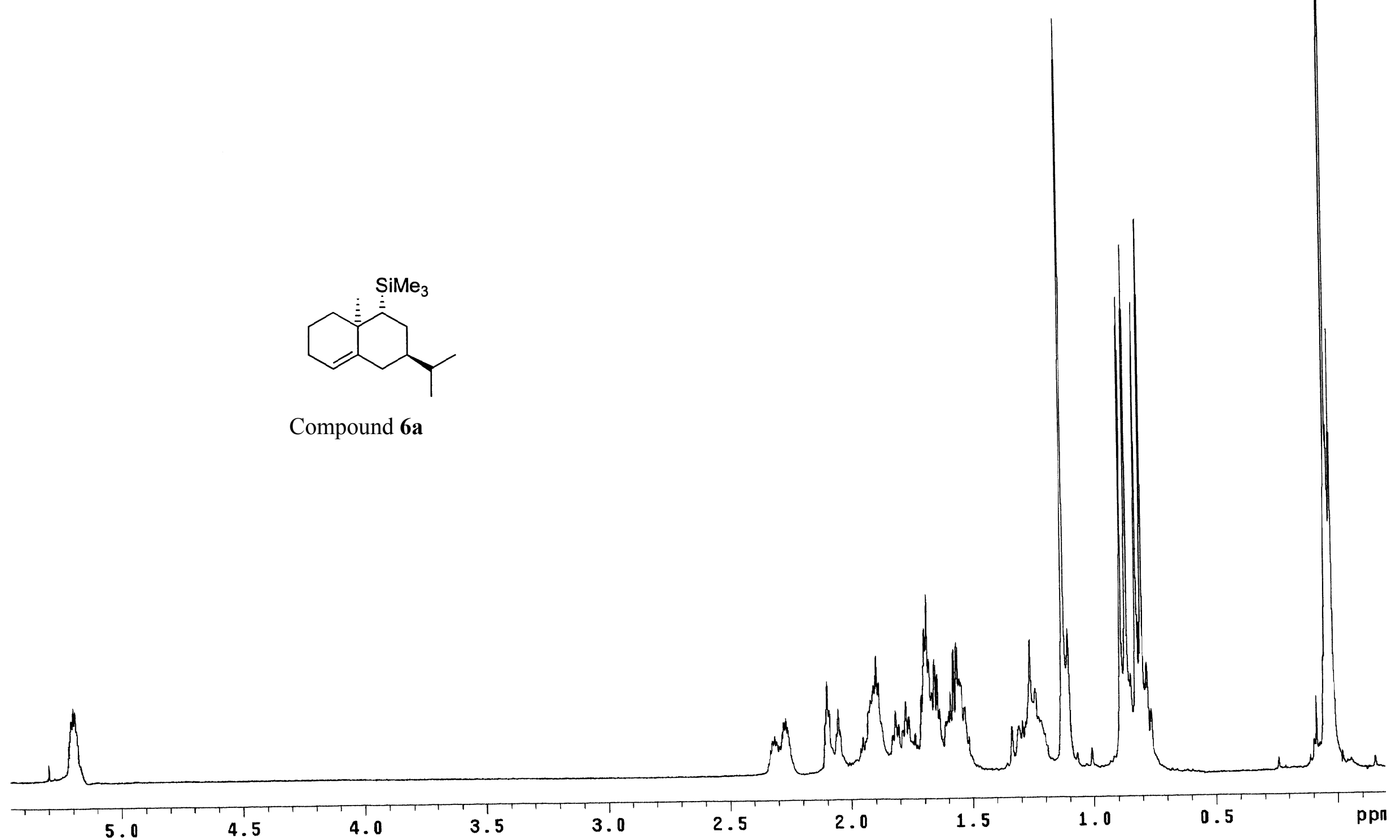




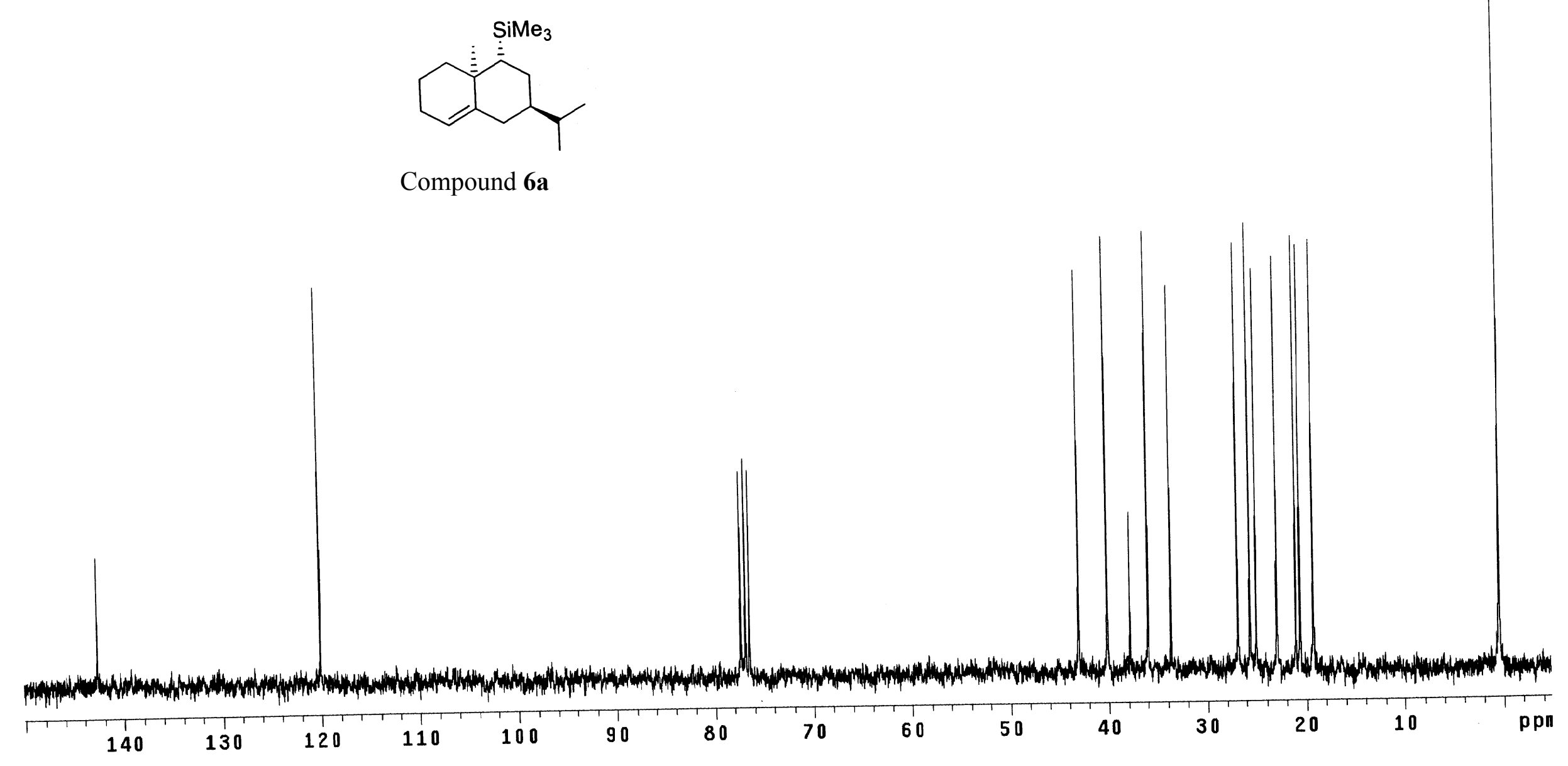




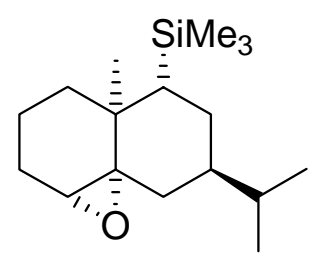

Compound 7a

$$
7
$$




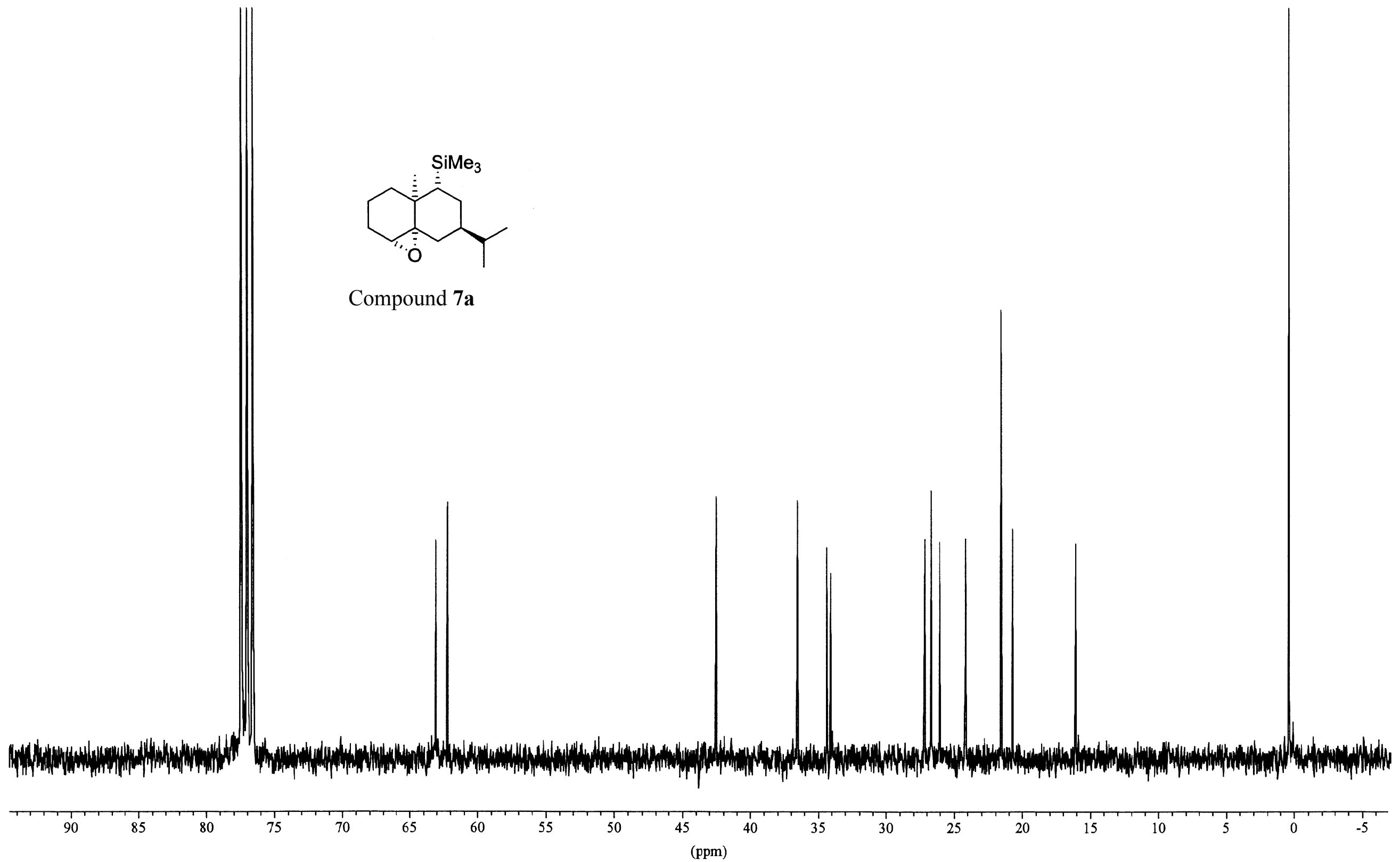




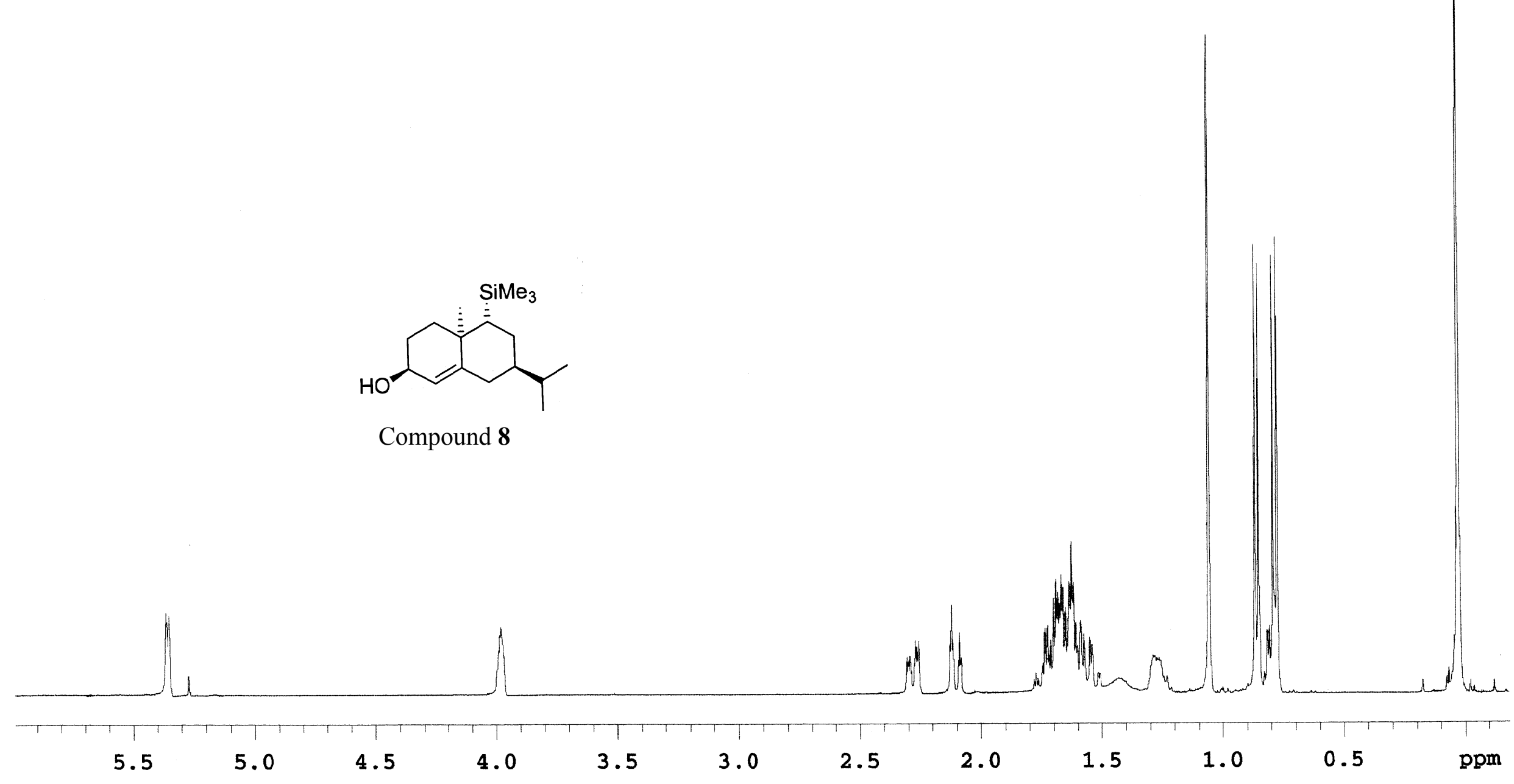




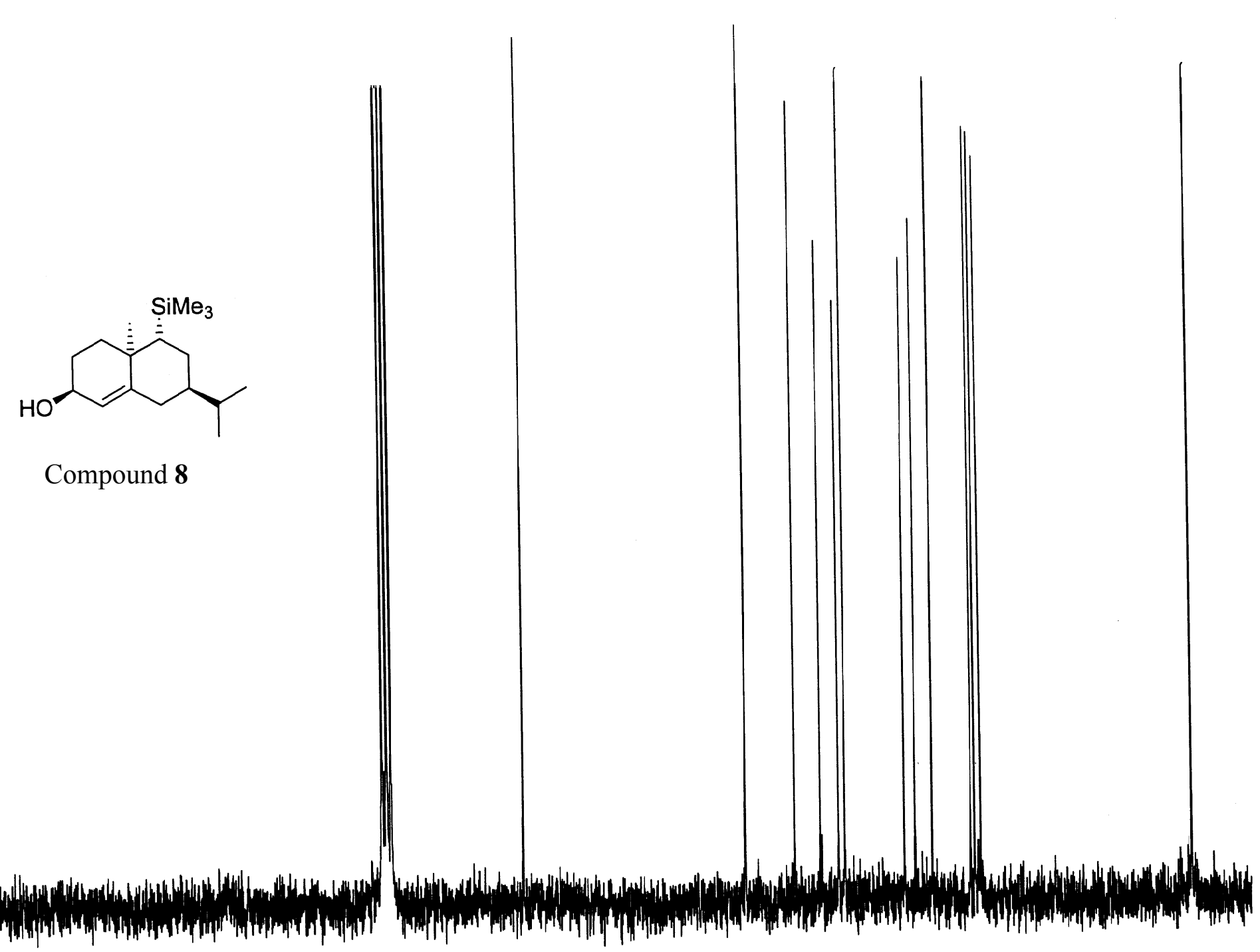

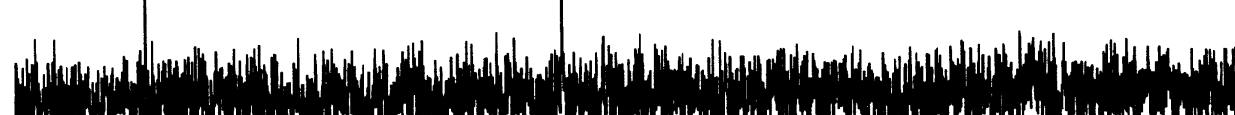

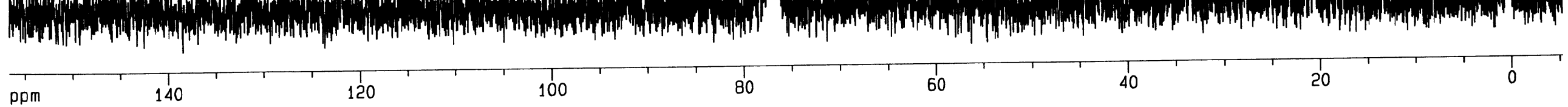




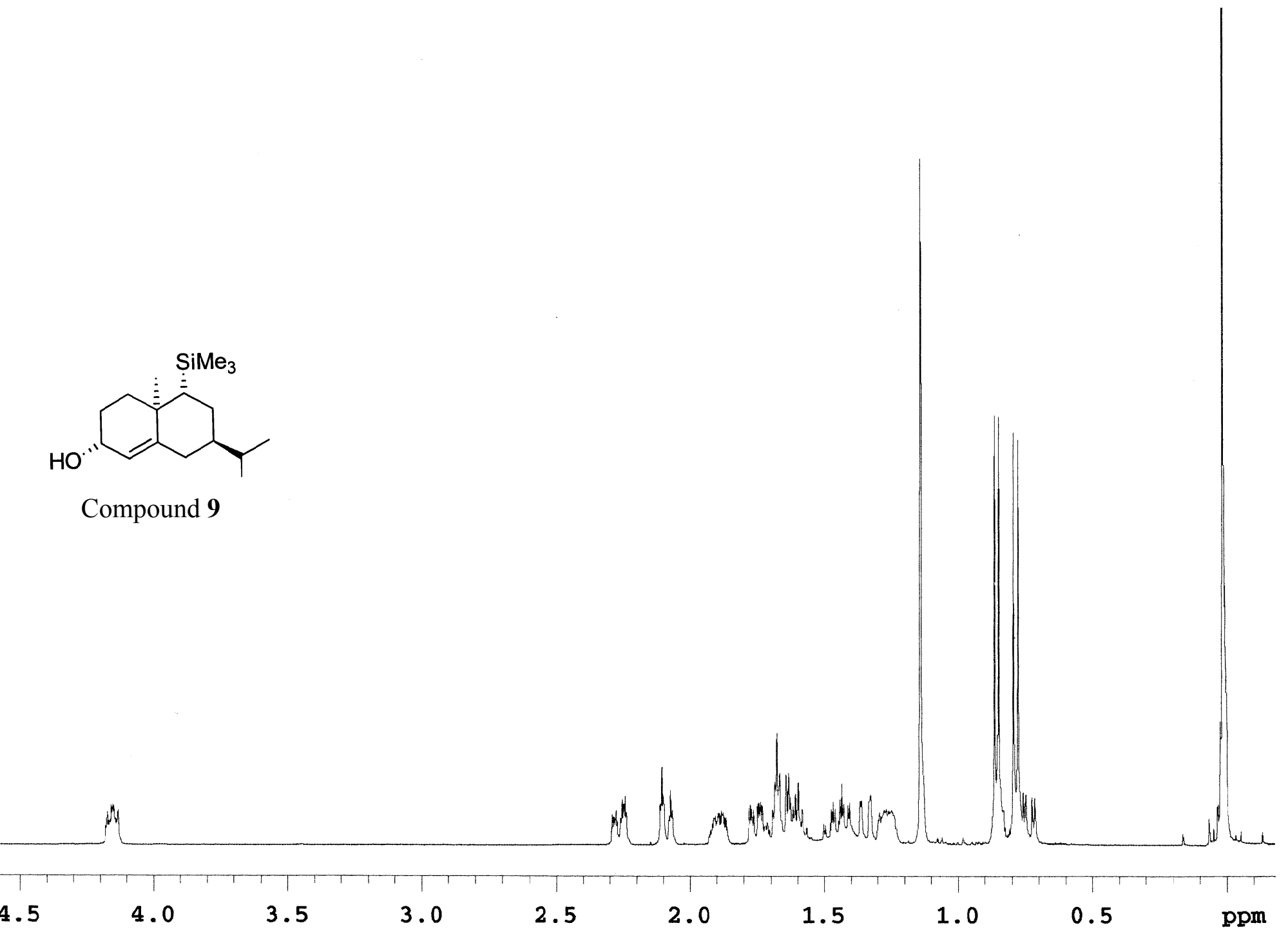





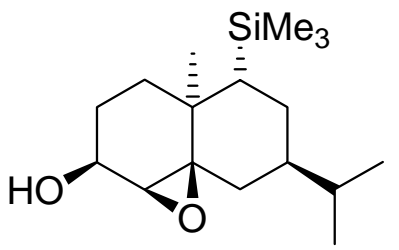

Compound 10

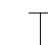




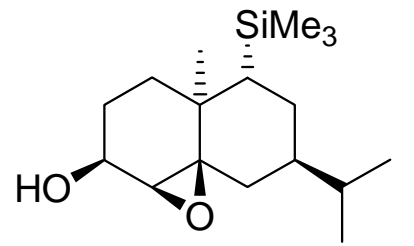

Compound 10

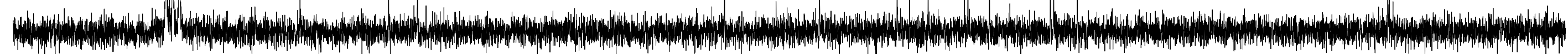




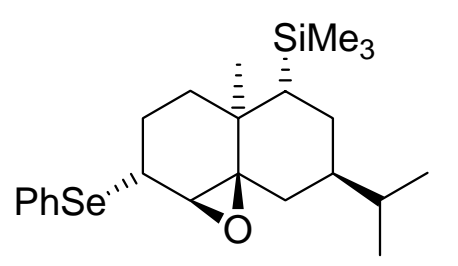

Compound 11

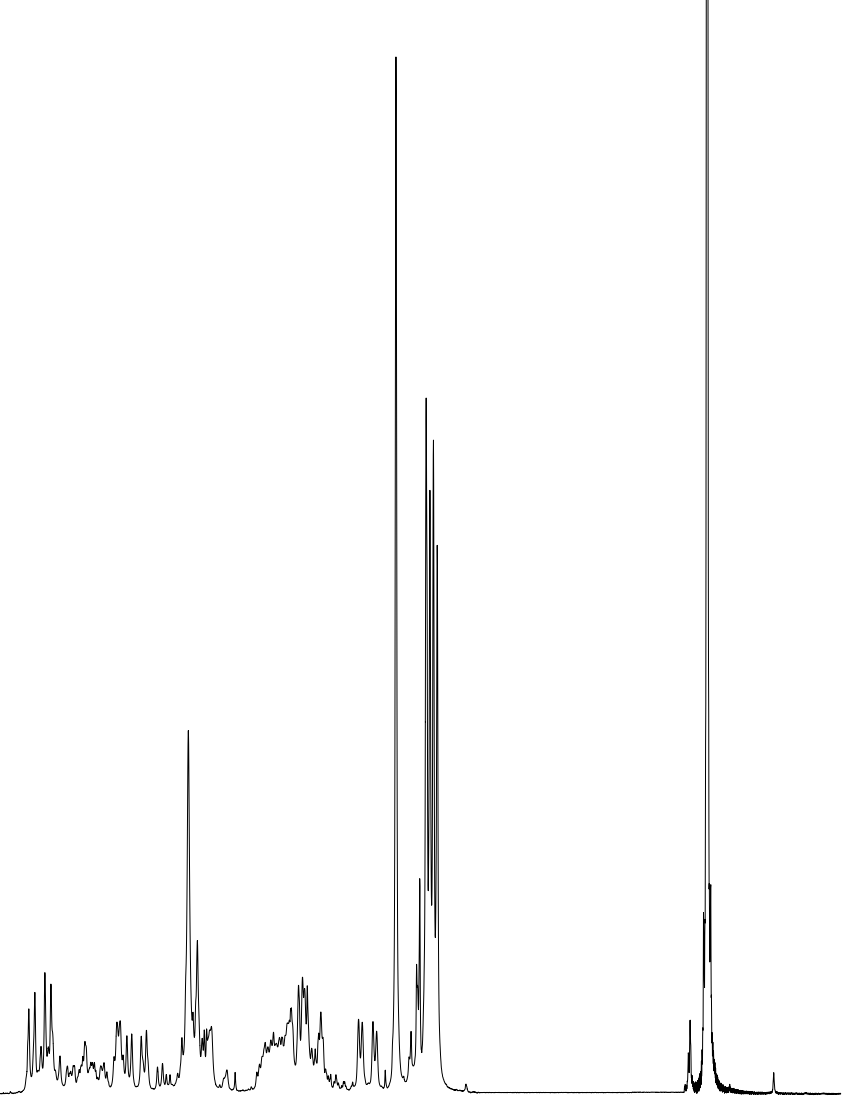




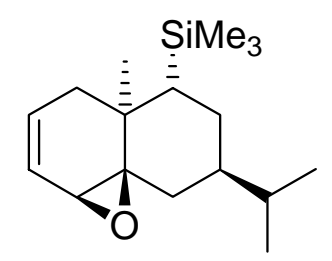

Compound 12

H

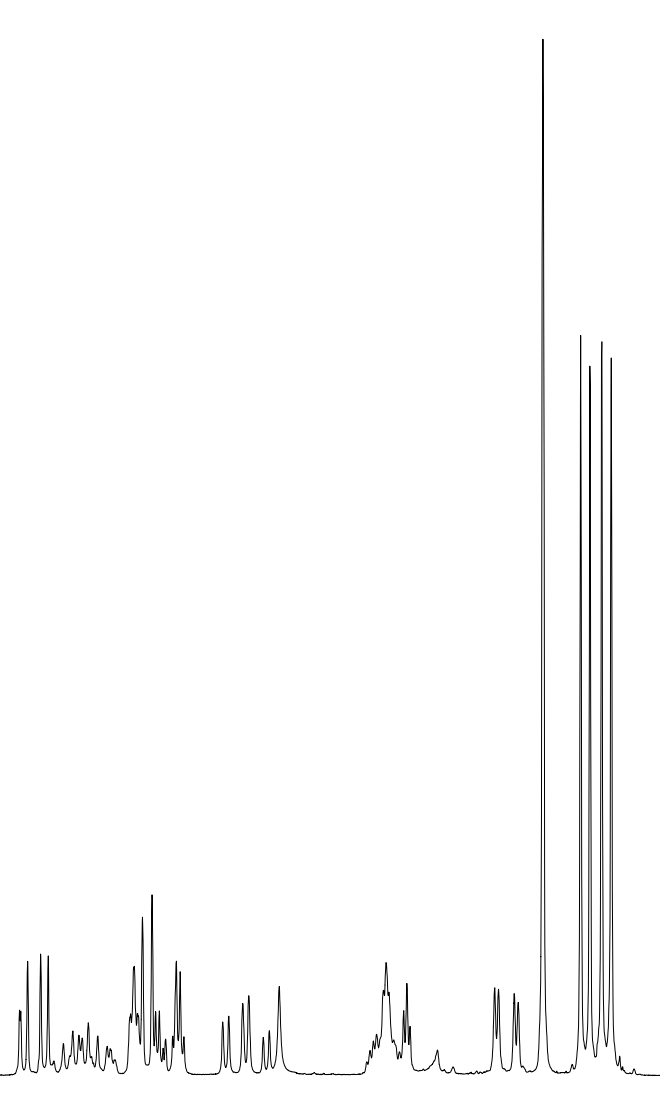


S-

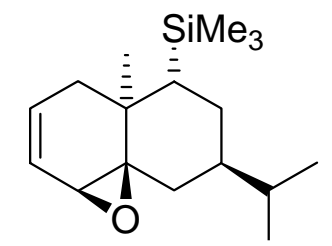

Compound 12 


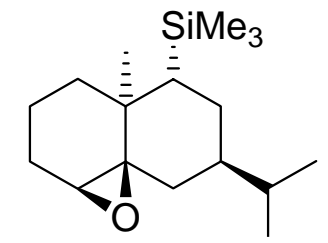

Compound 13 


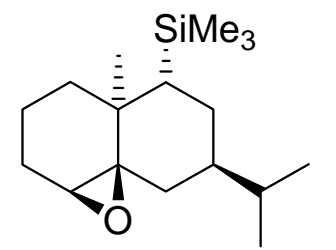

Compound 13

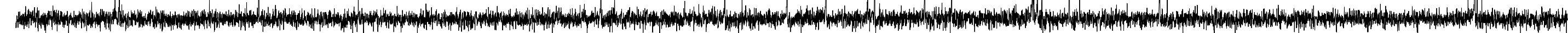




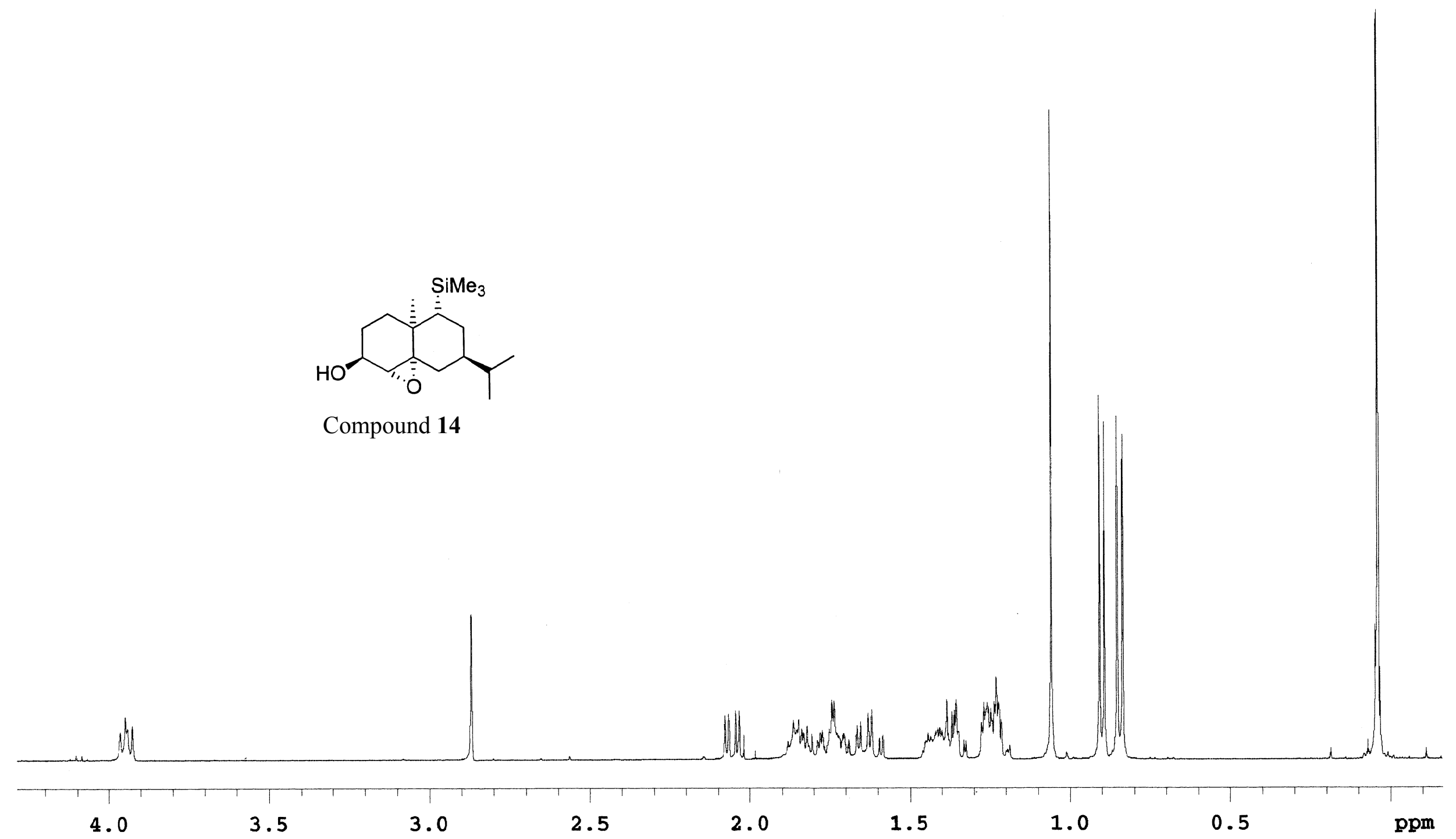


S-
27

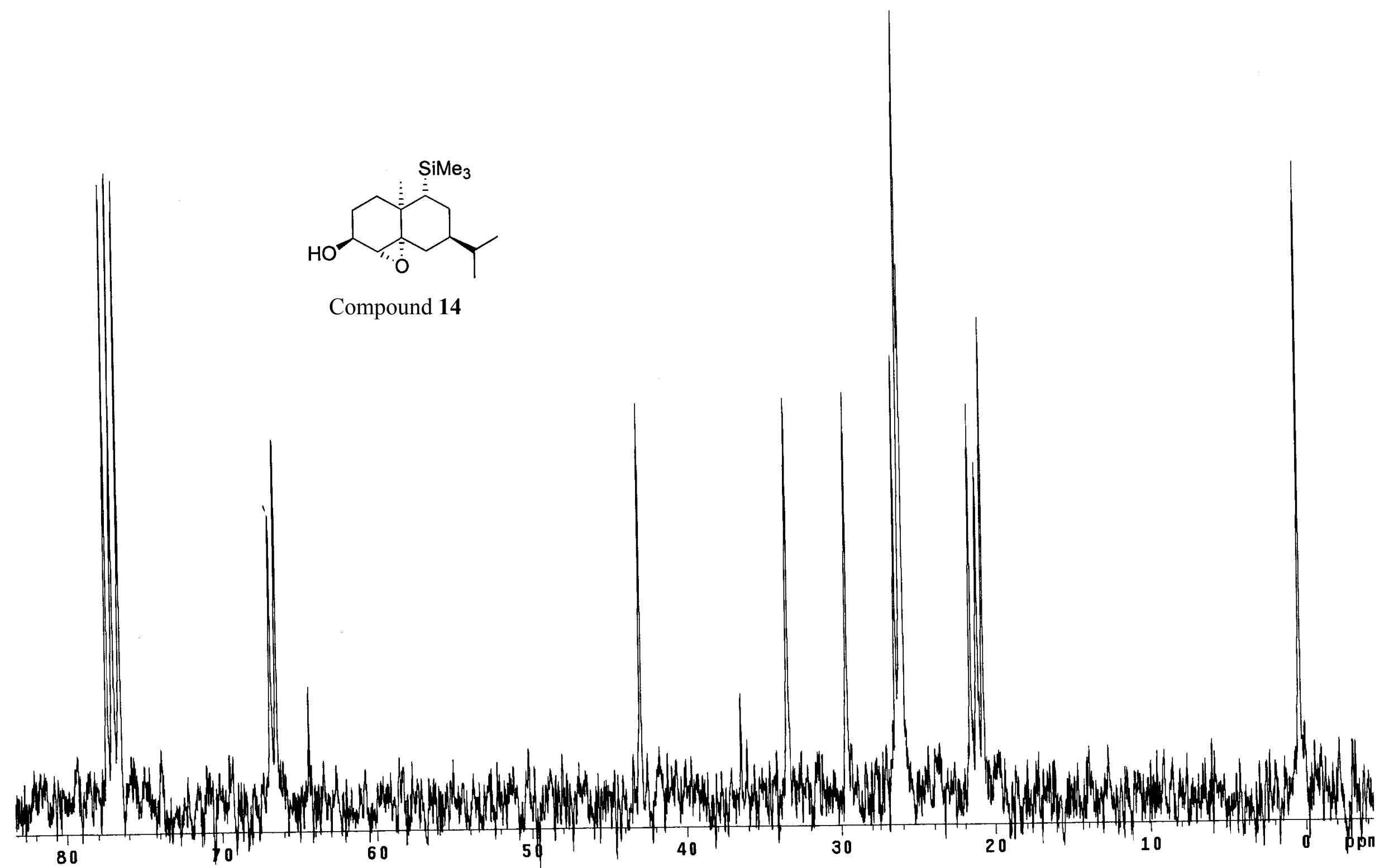




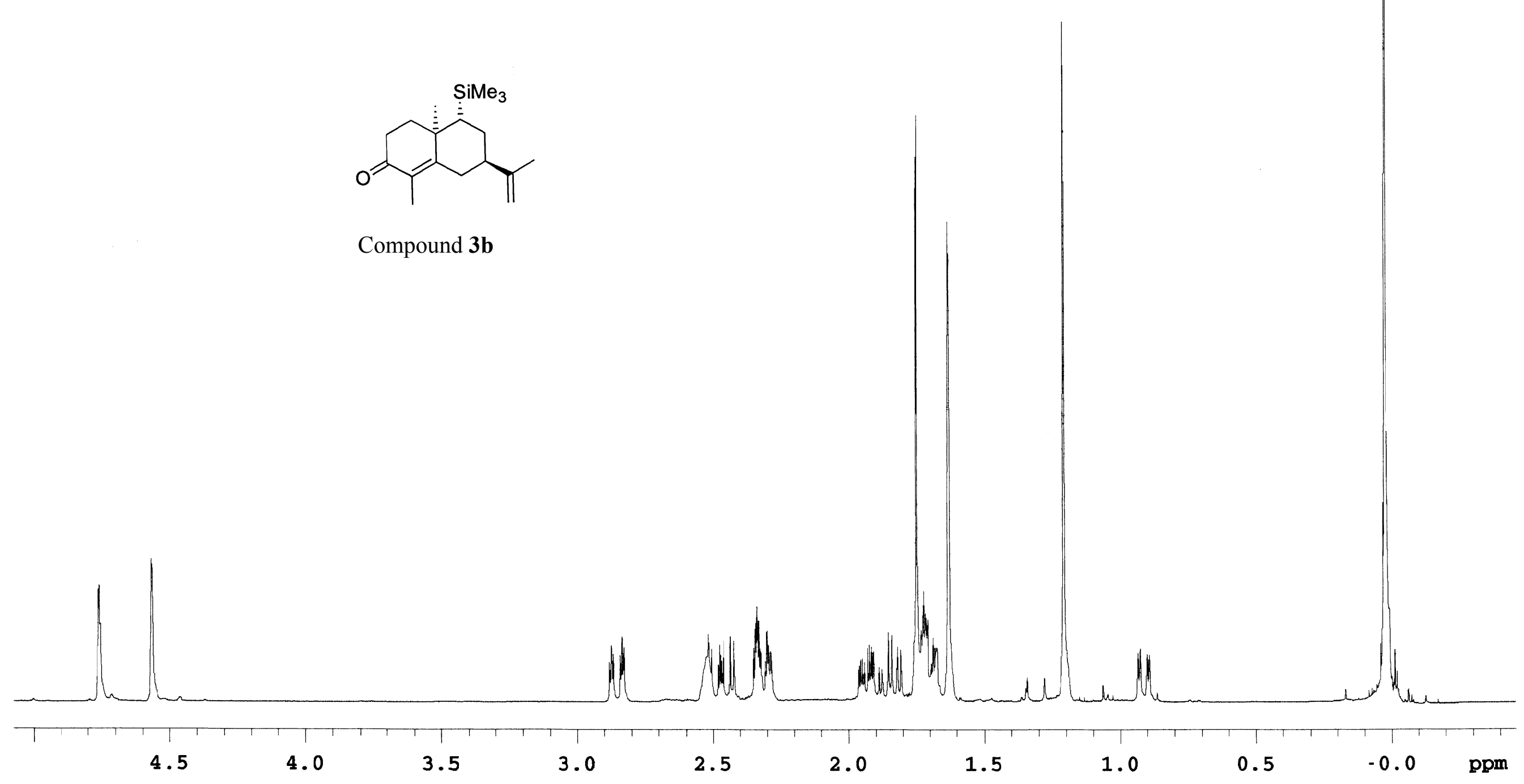




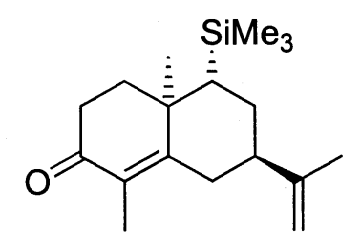

Compound 3b

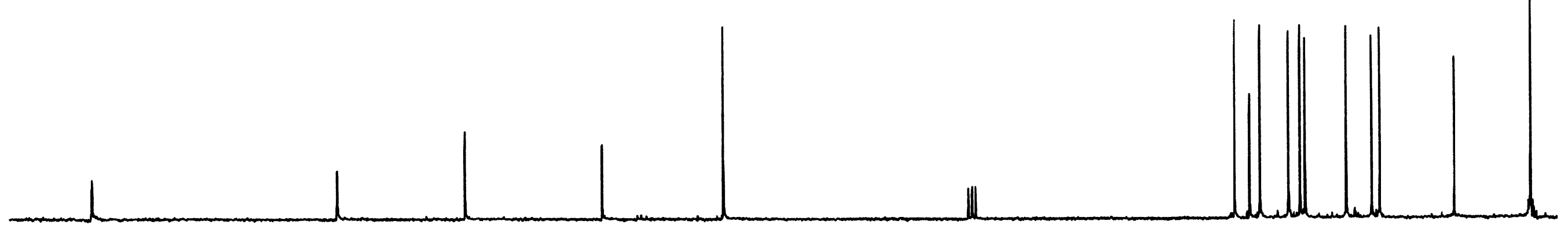


S-
30

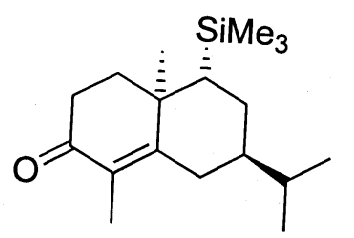

Compound 4b

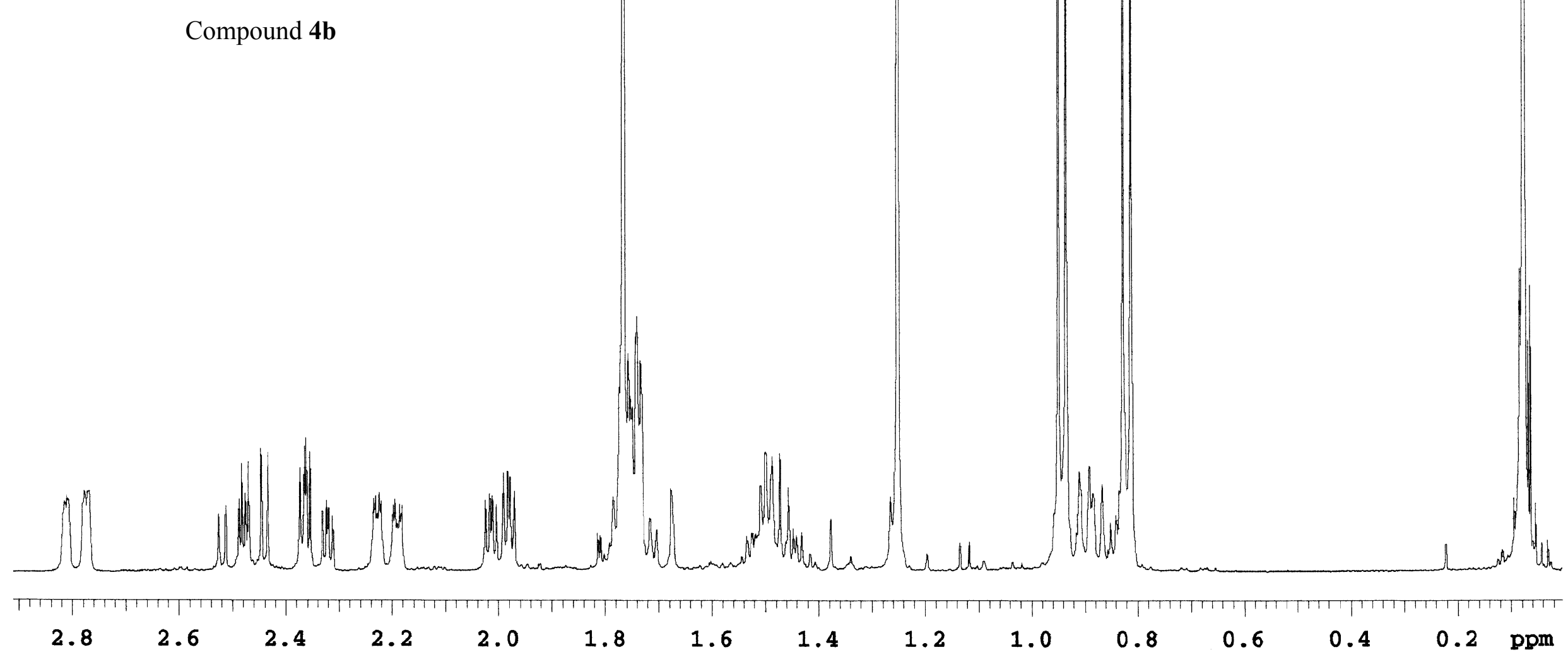




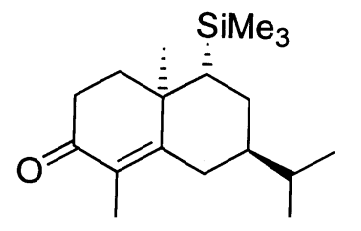

Compound 4b

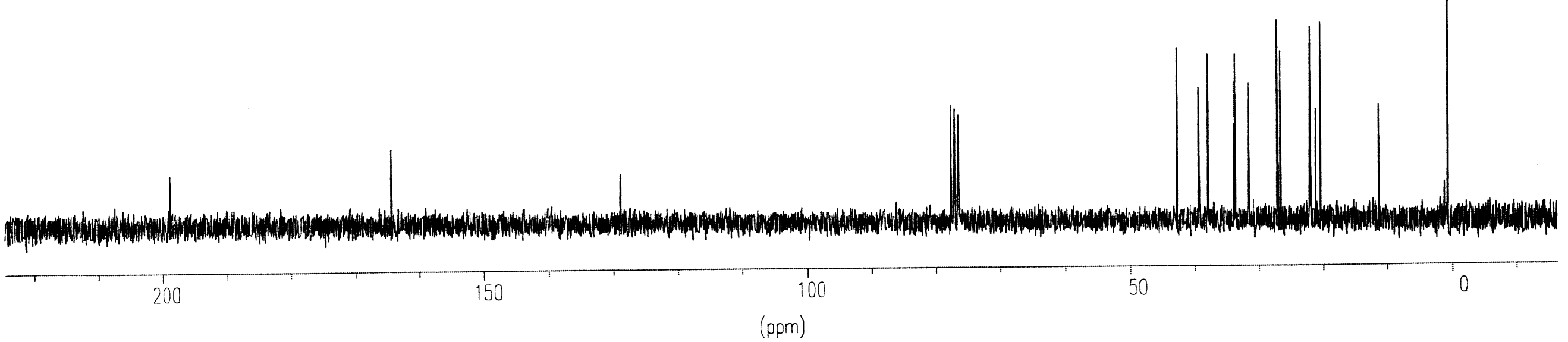


S-
32

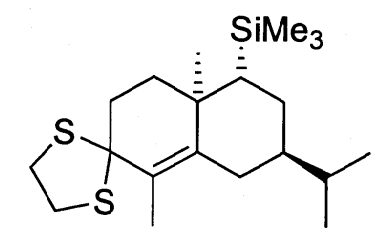

Compound $\mathbf{5 b}$

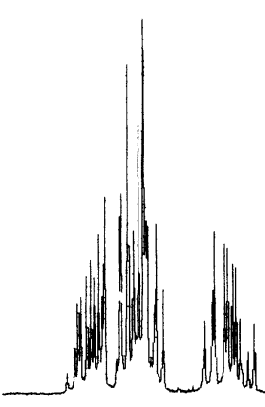

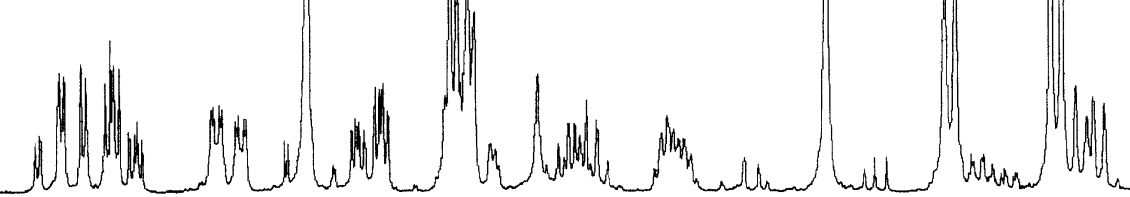

2.5

2.0

1.5

1.0

0.5 


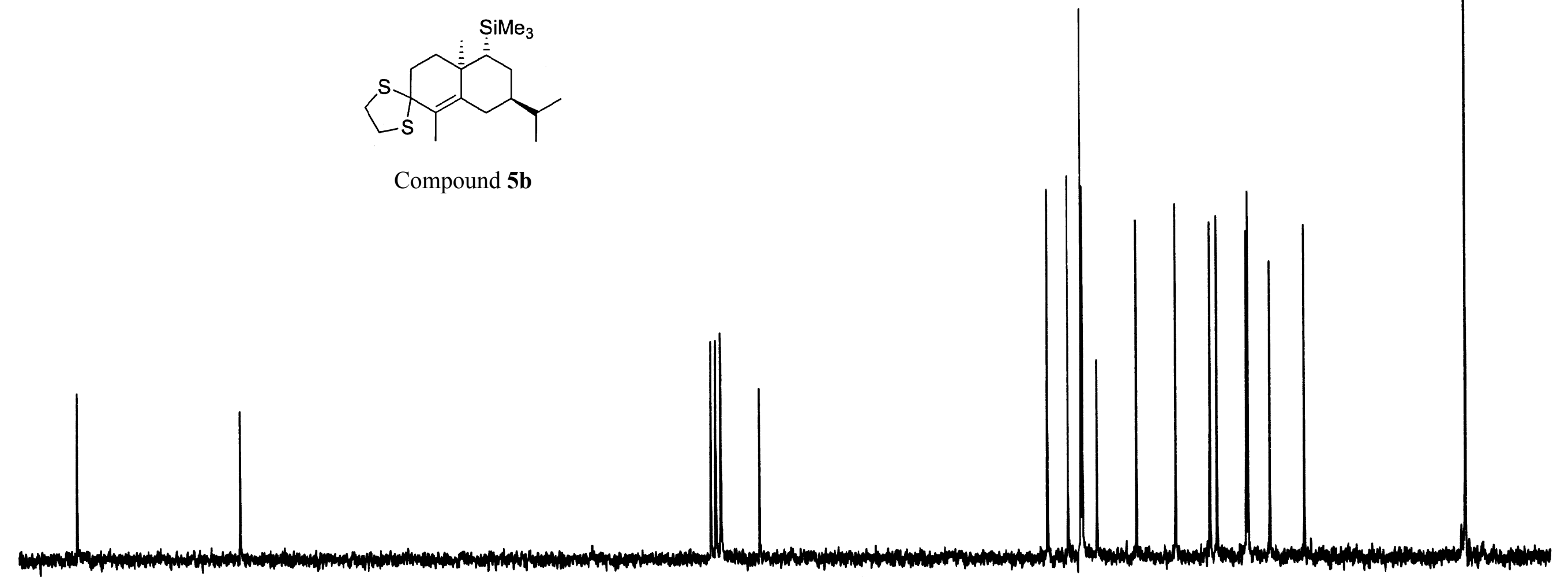

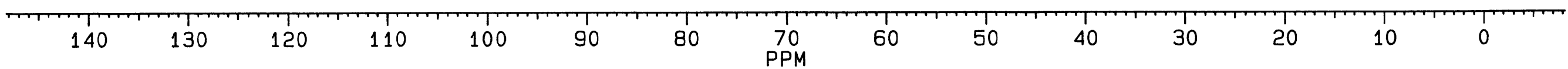




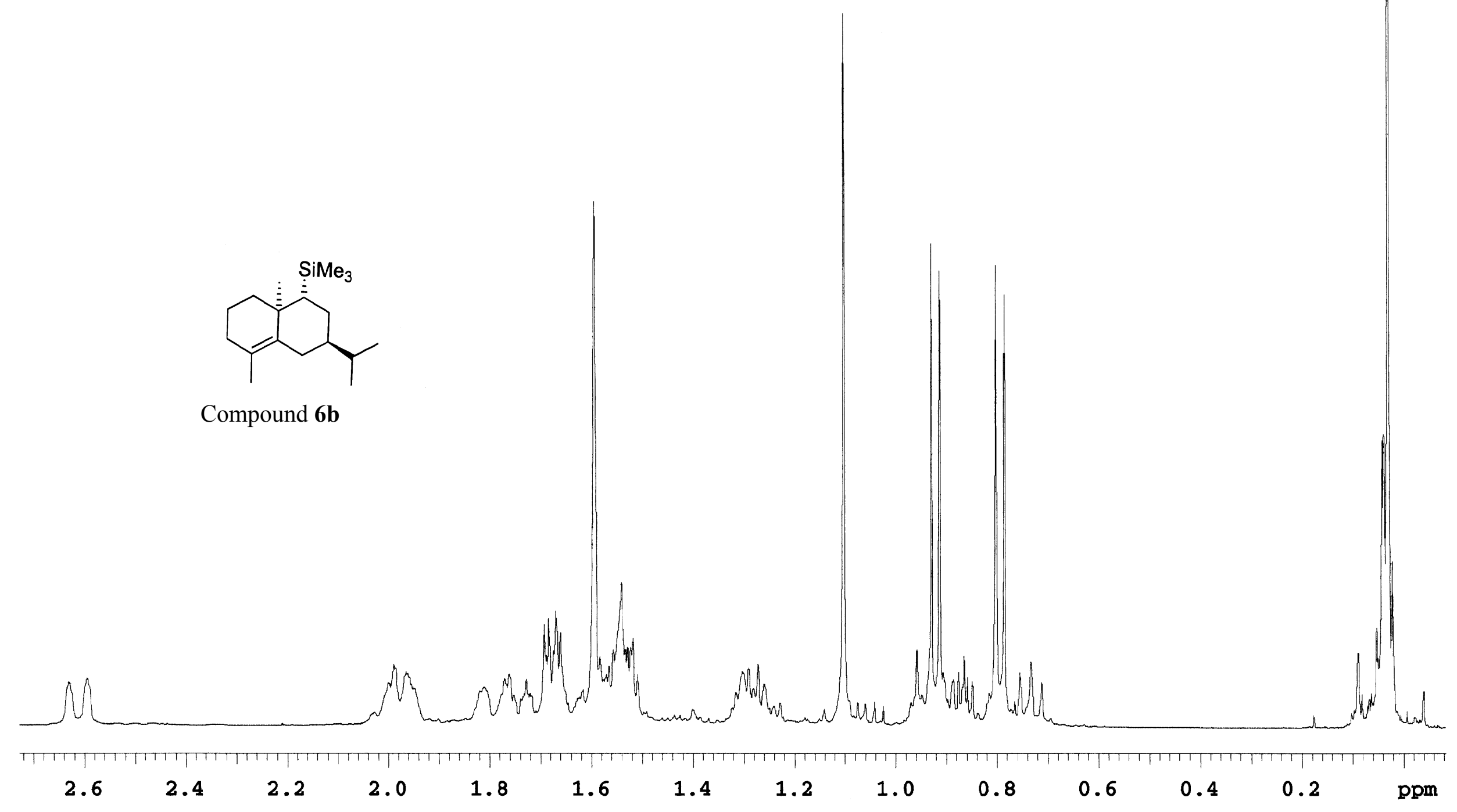




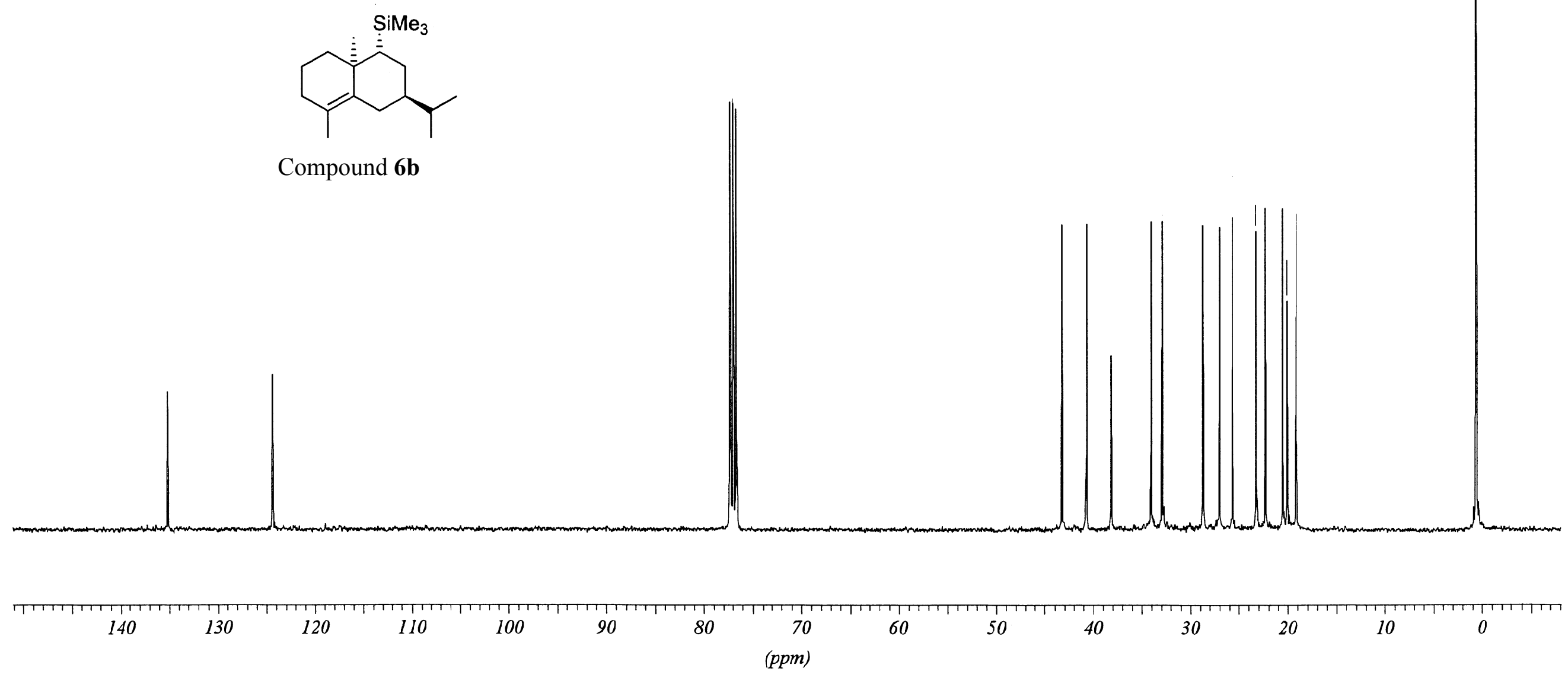


S-
36

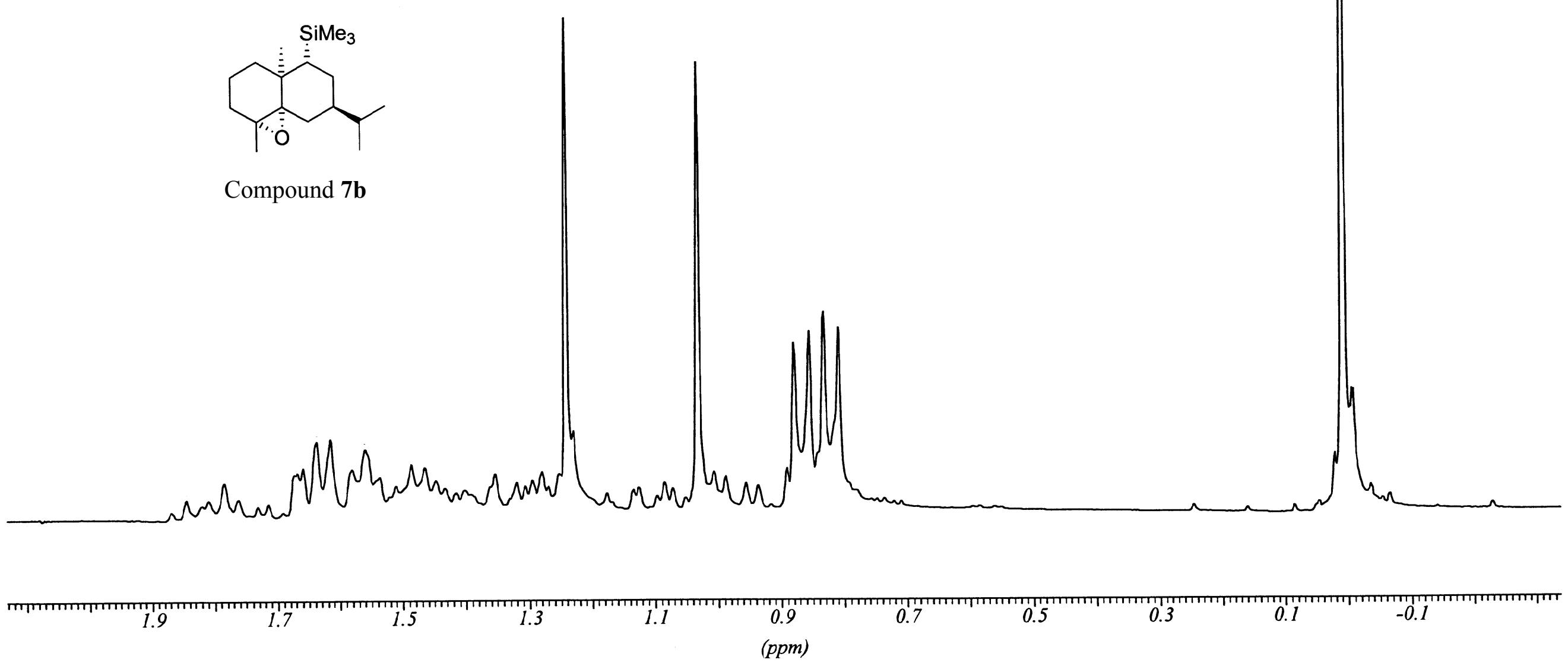


S-
37

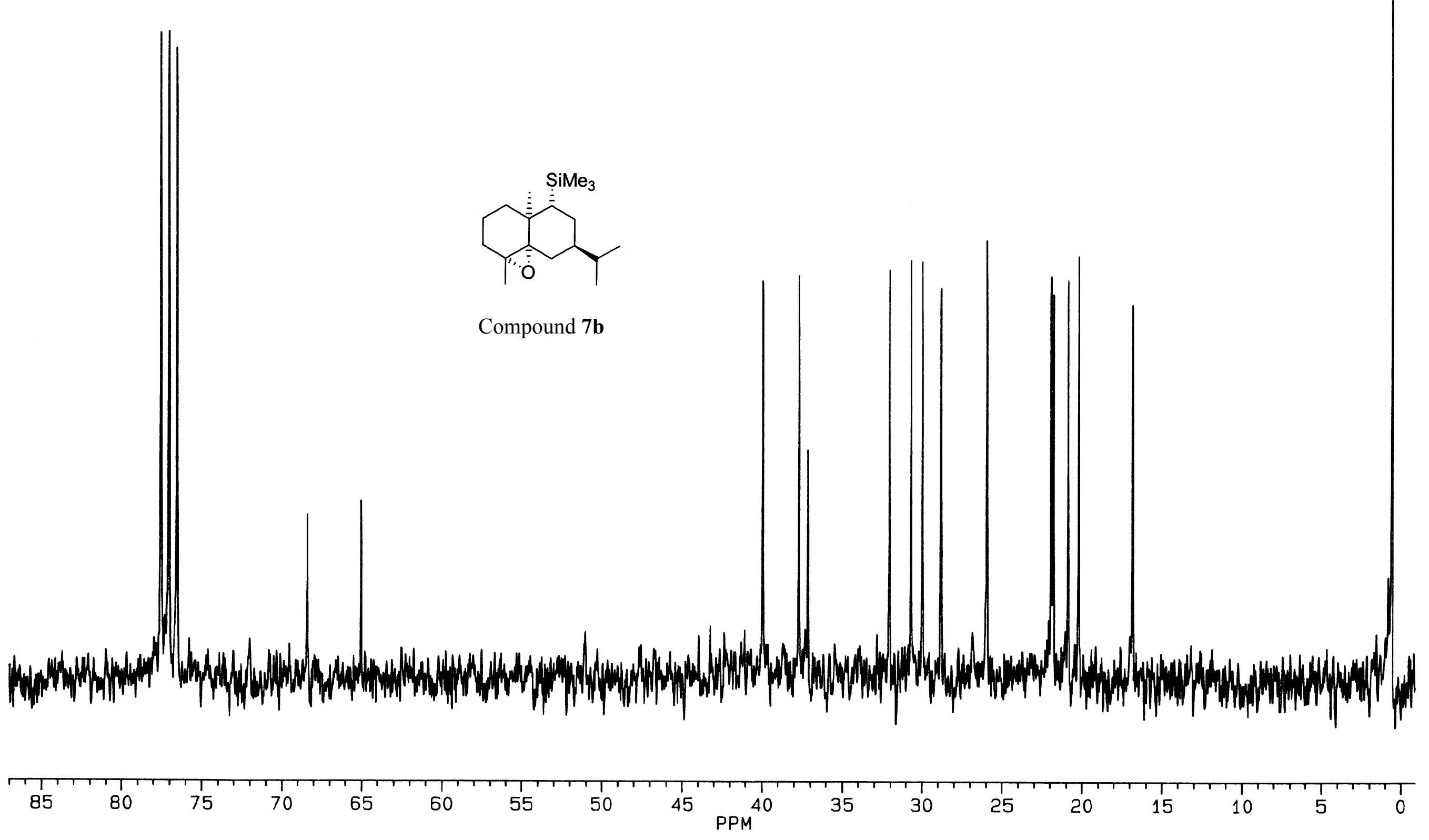




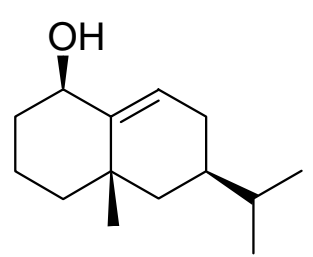

Compound 15

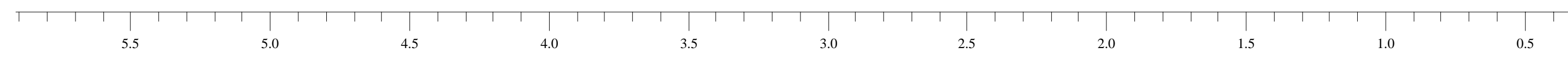


S-

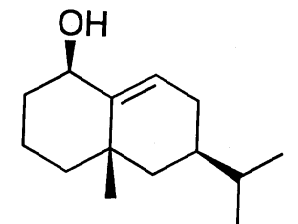

Compound 15

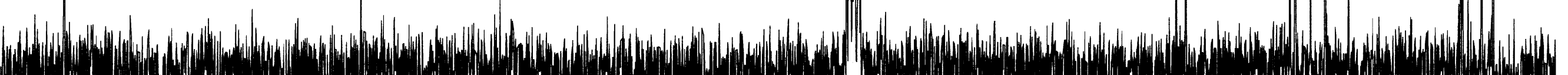

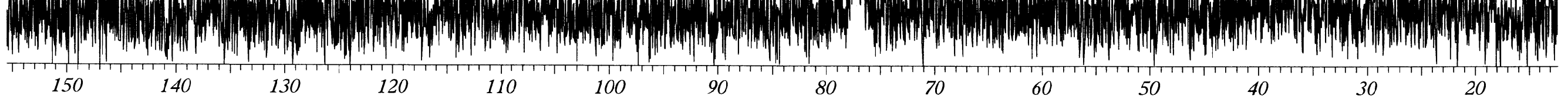
(ppm) 


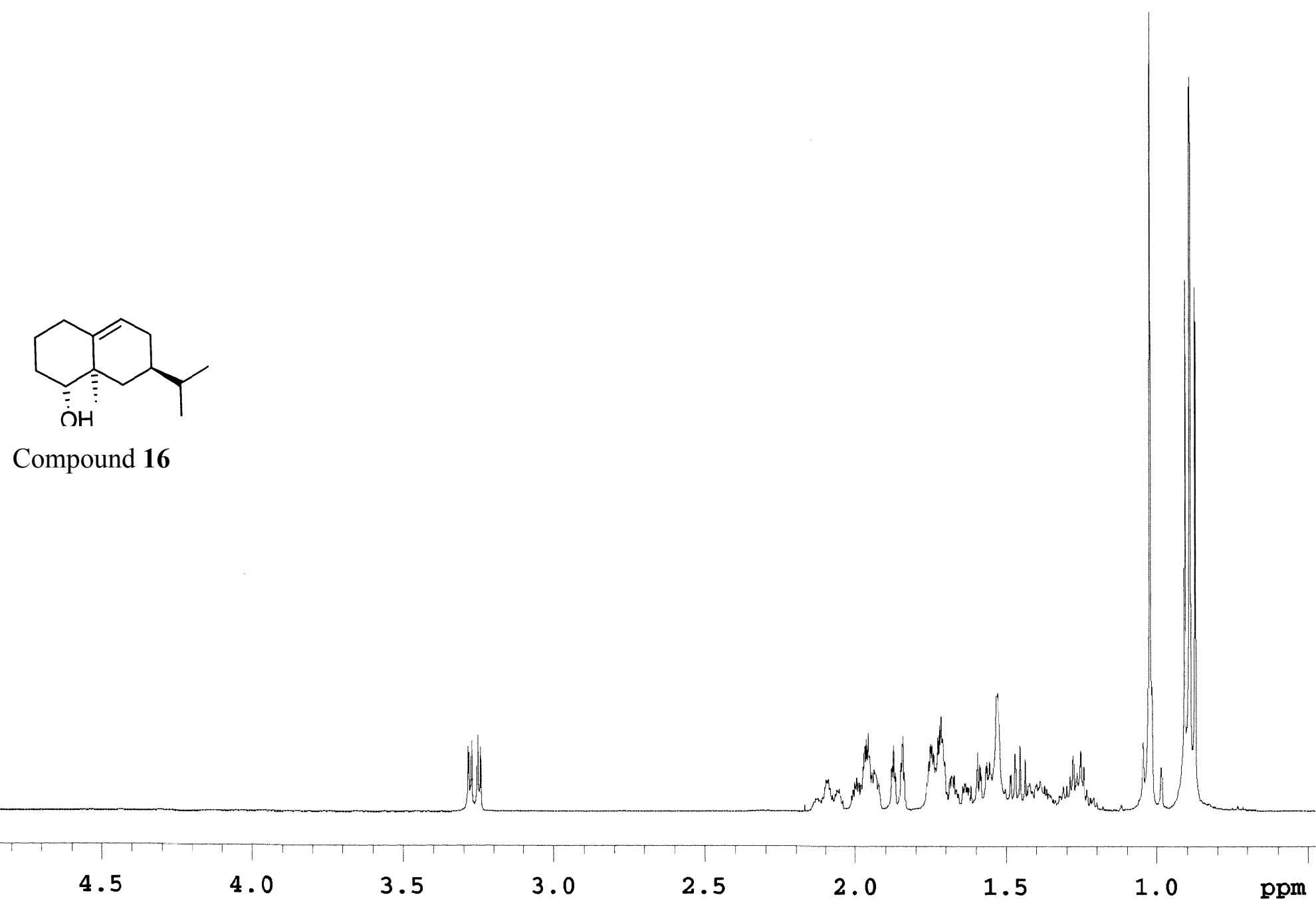




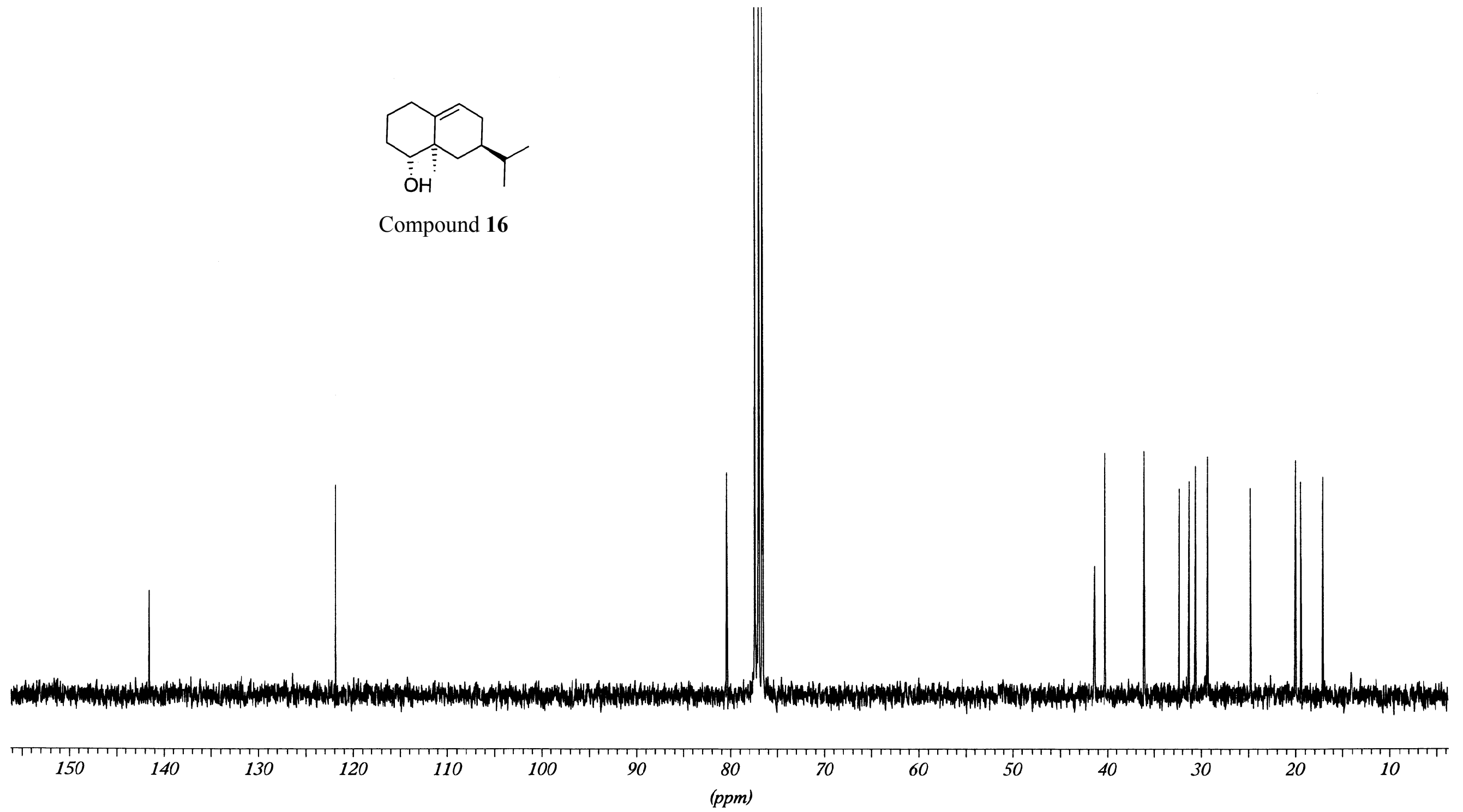




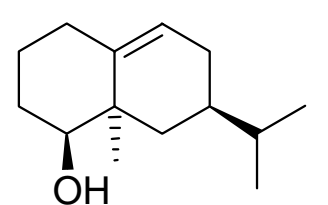

Compound 17

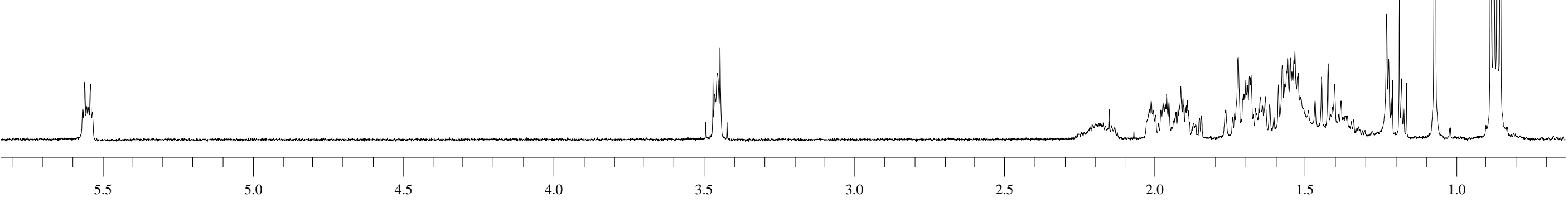


S-

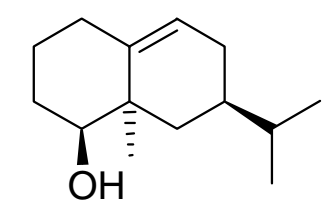

Compound 17

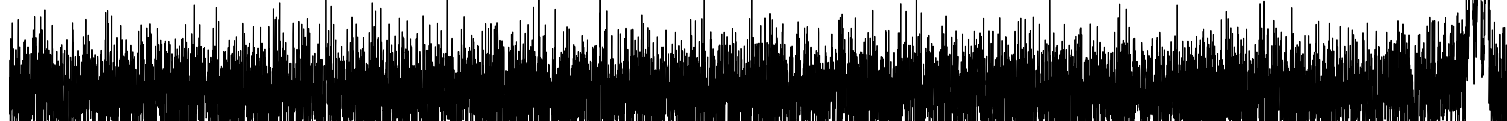

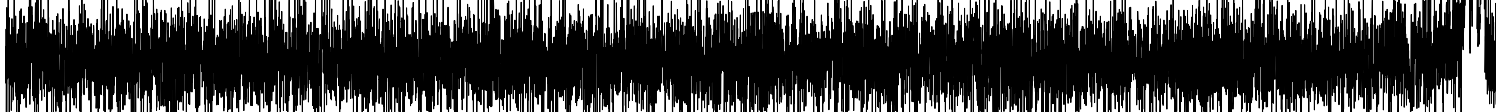

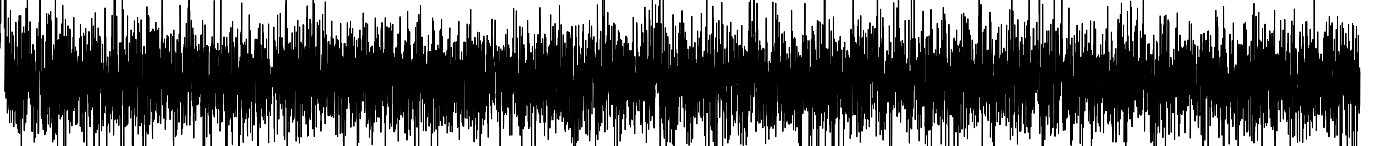




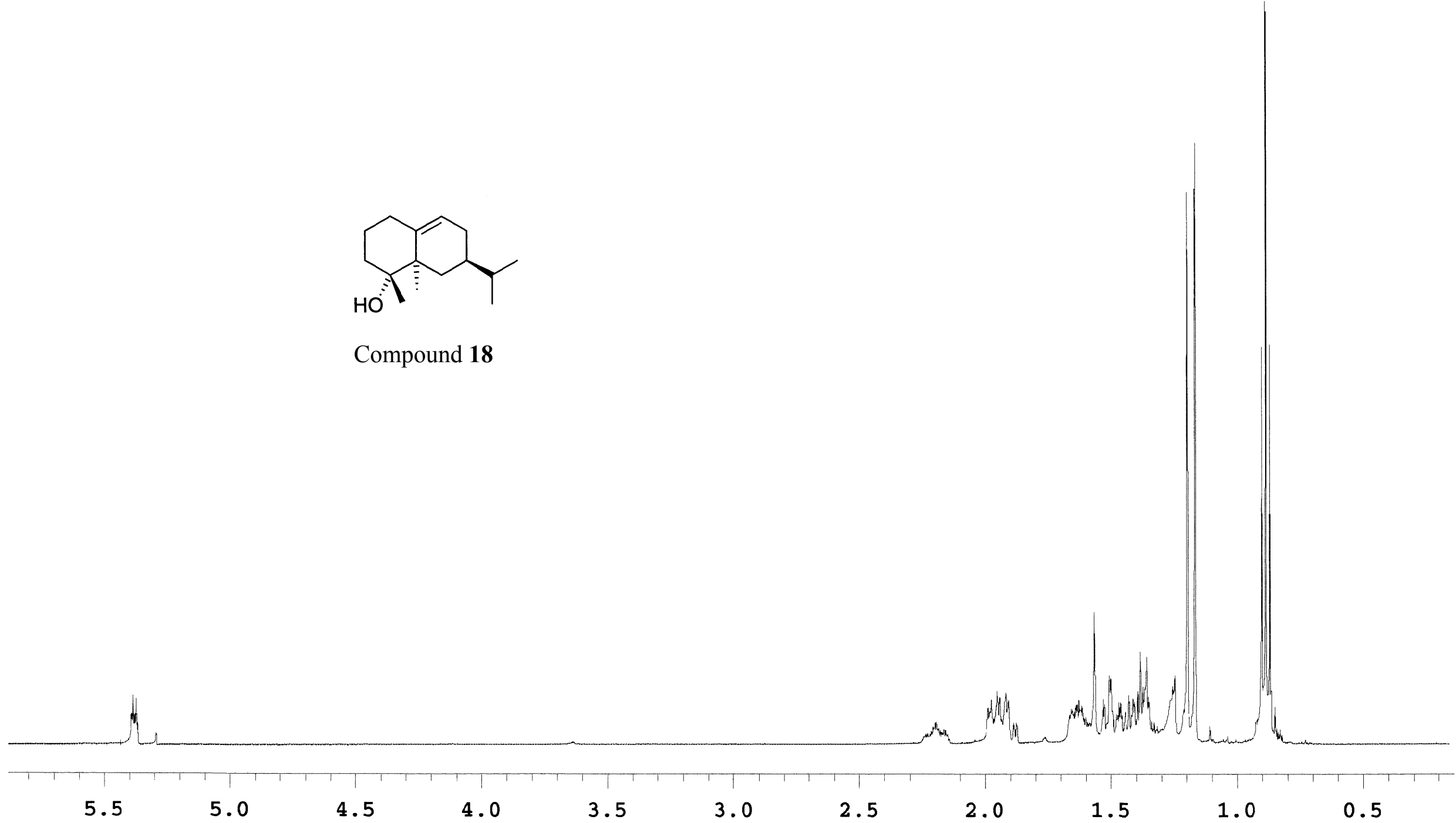


45

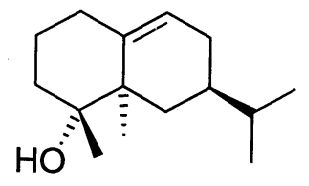

Compound 18

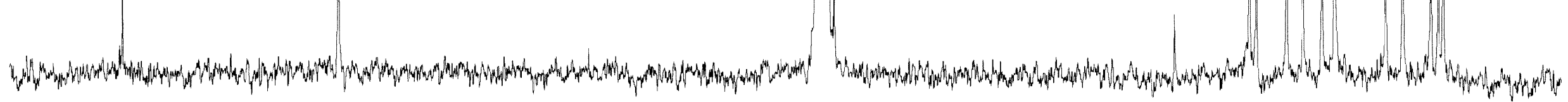

$\begin{array}{llllllllllllll}140 & 130 & 120 & 110 & 100 & 90 & 80 & 70 & 60 & 50 & 40 & 30 & 20 & \text { ppm }\end{array}$




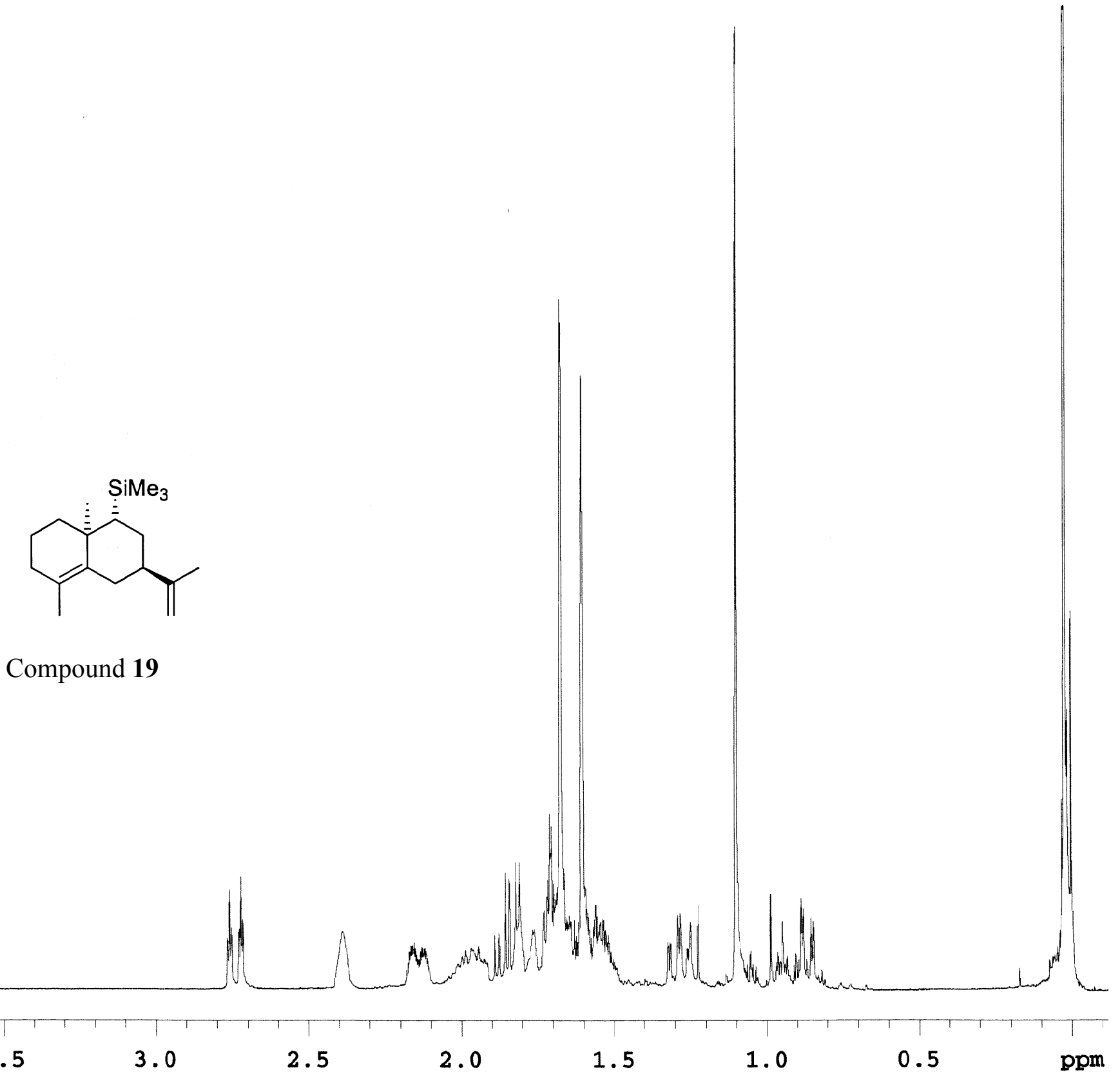




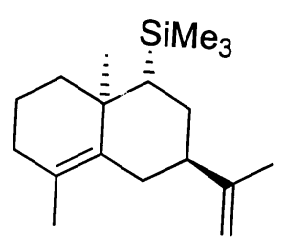

Compound 19

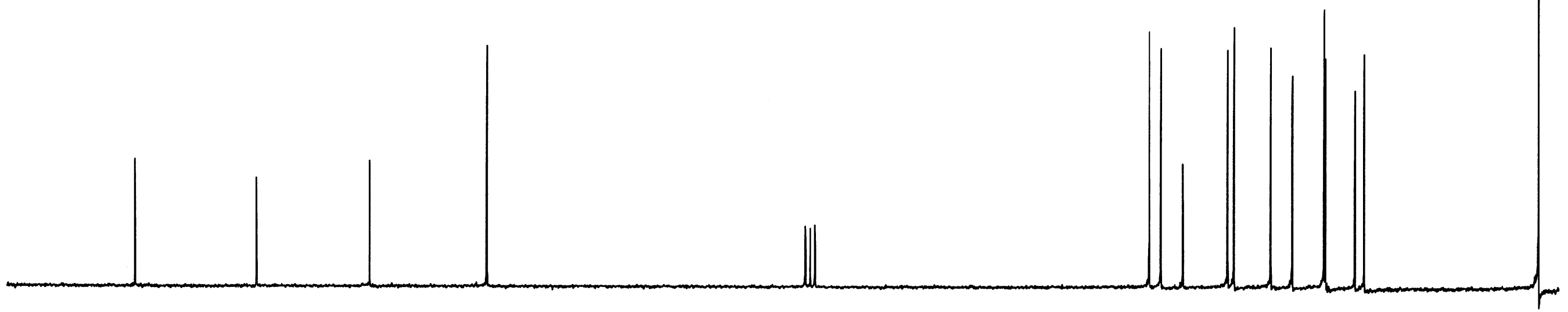




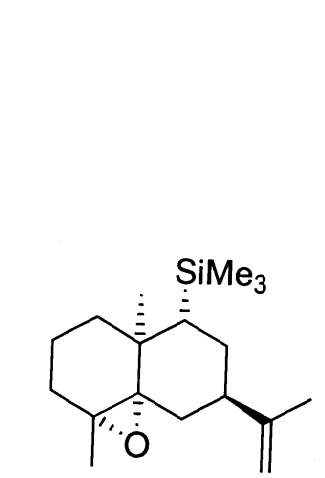

Compound 20

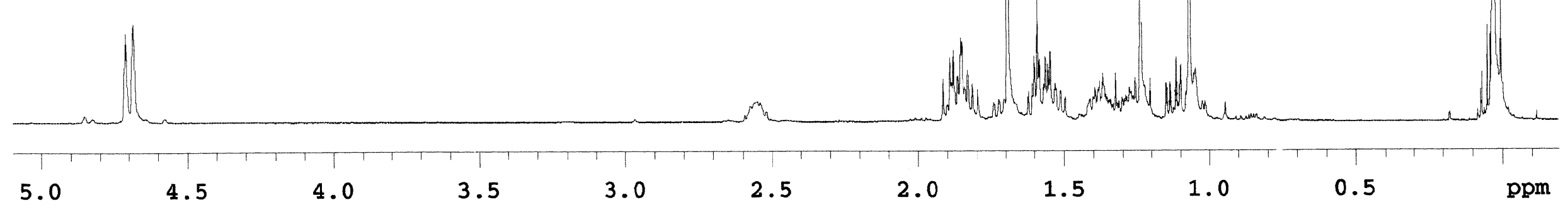




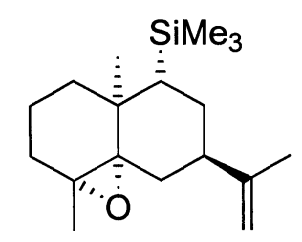

Compound 20

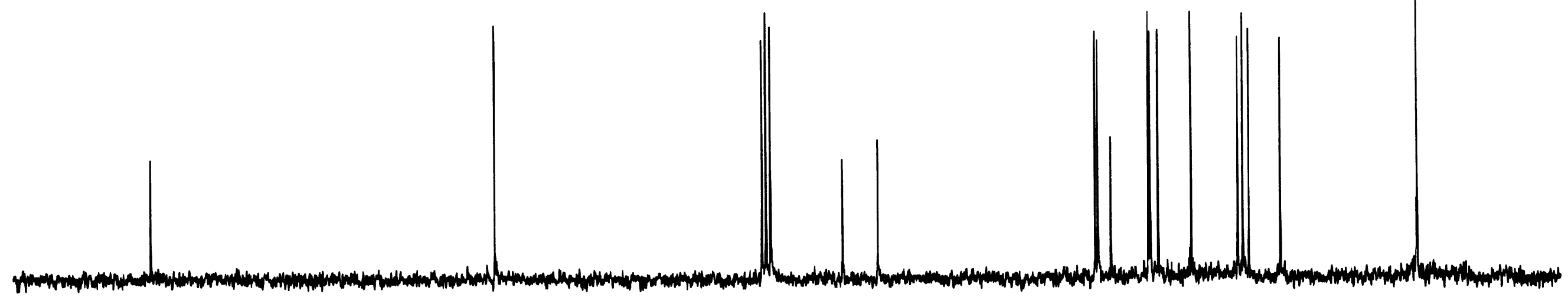




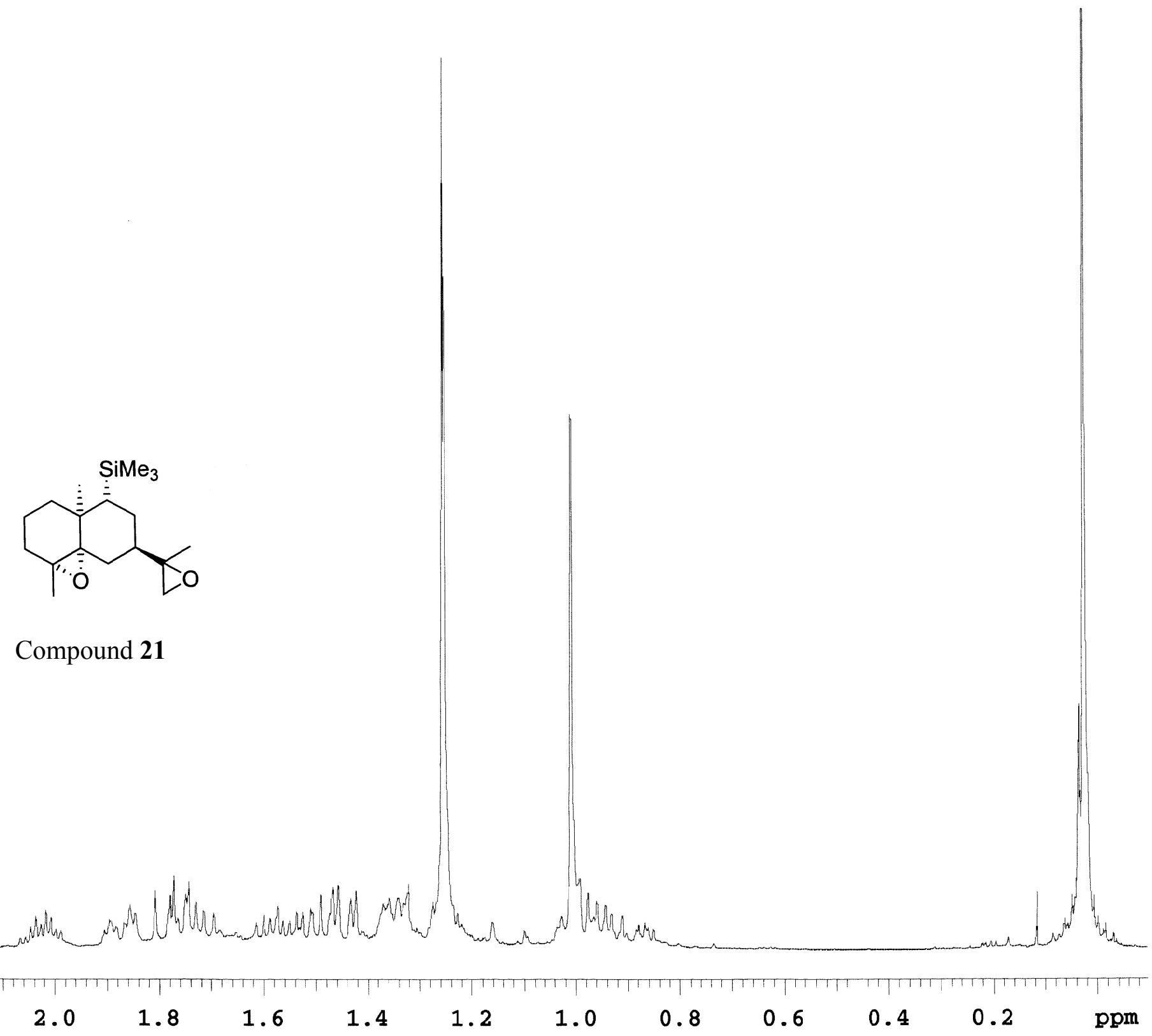


51

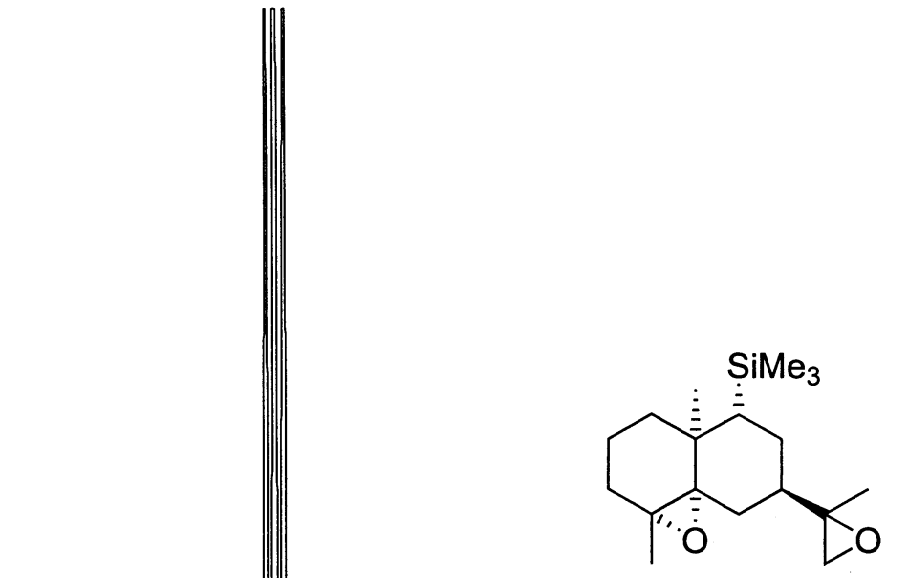

Compound 21

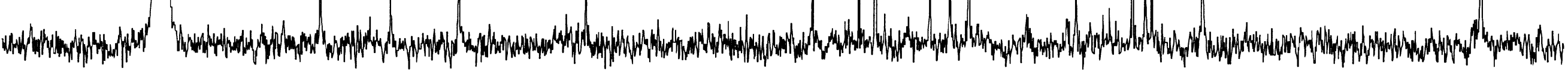




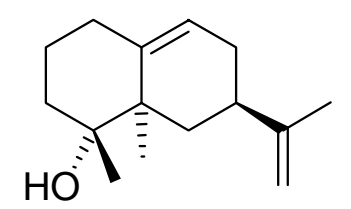

Compound 22

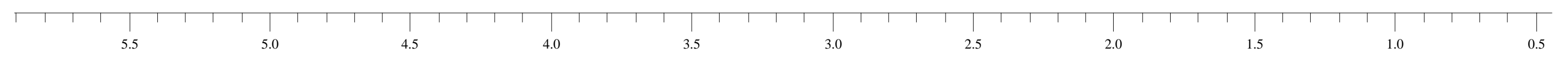




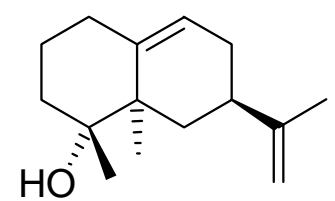

Compound 22 in DMSO- $d_{6}$

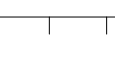




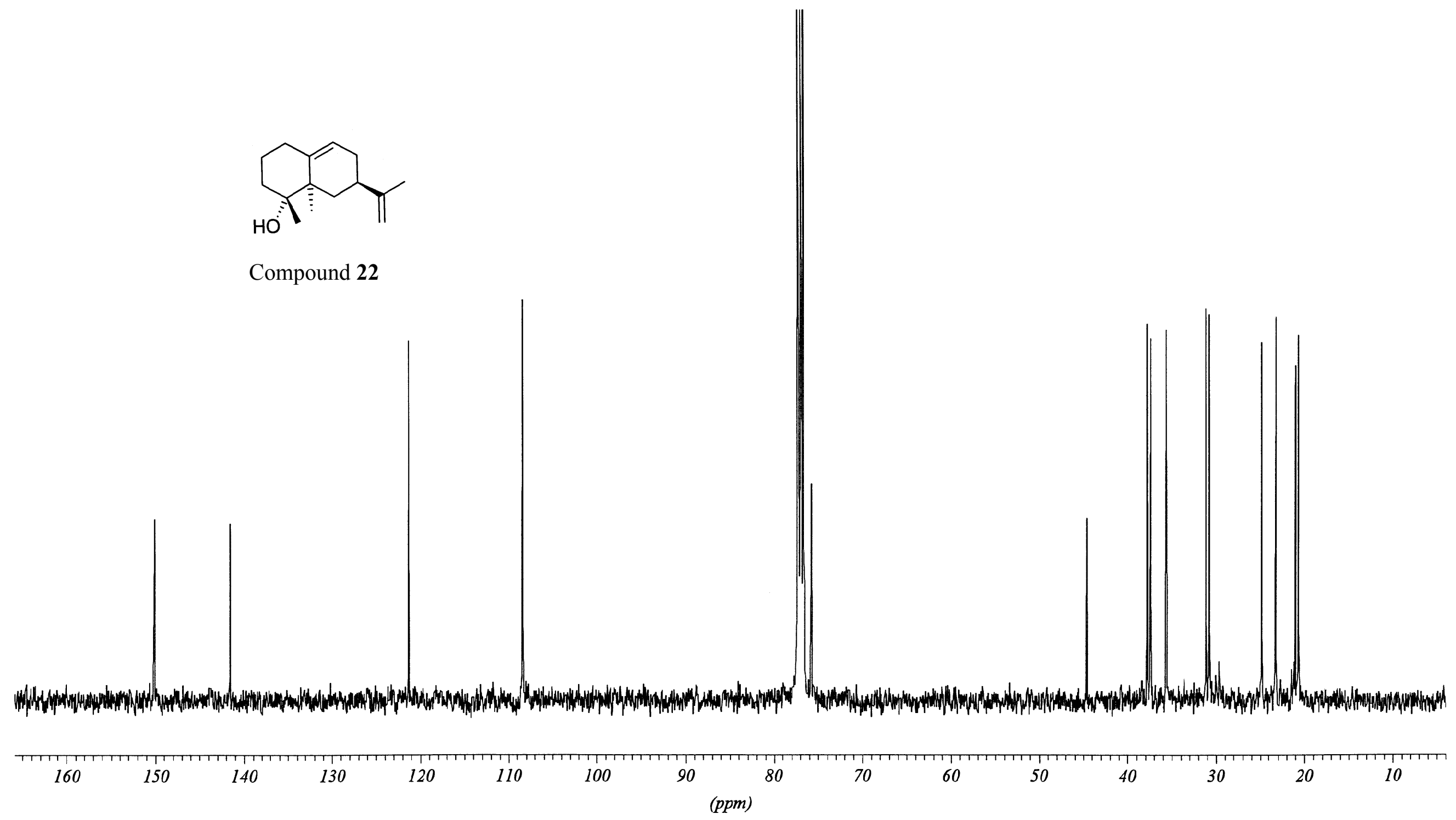


S-
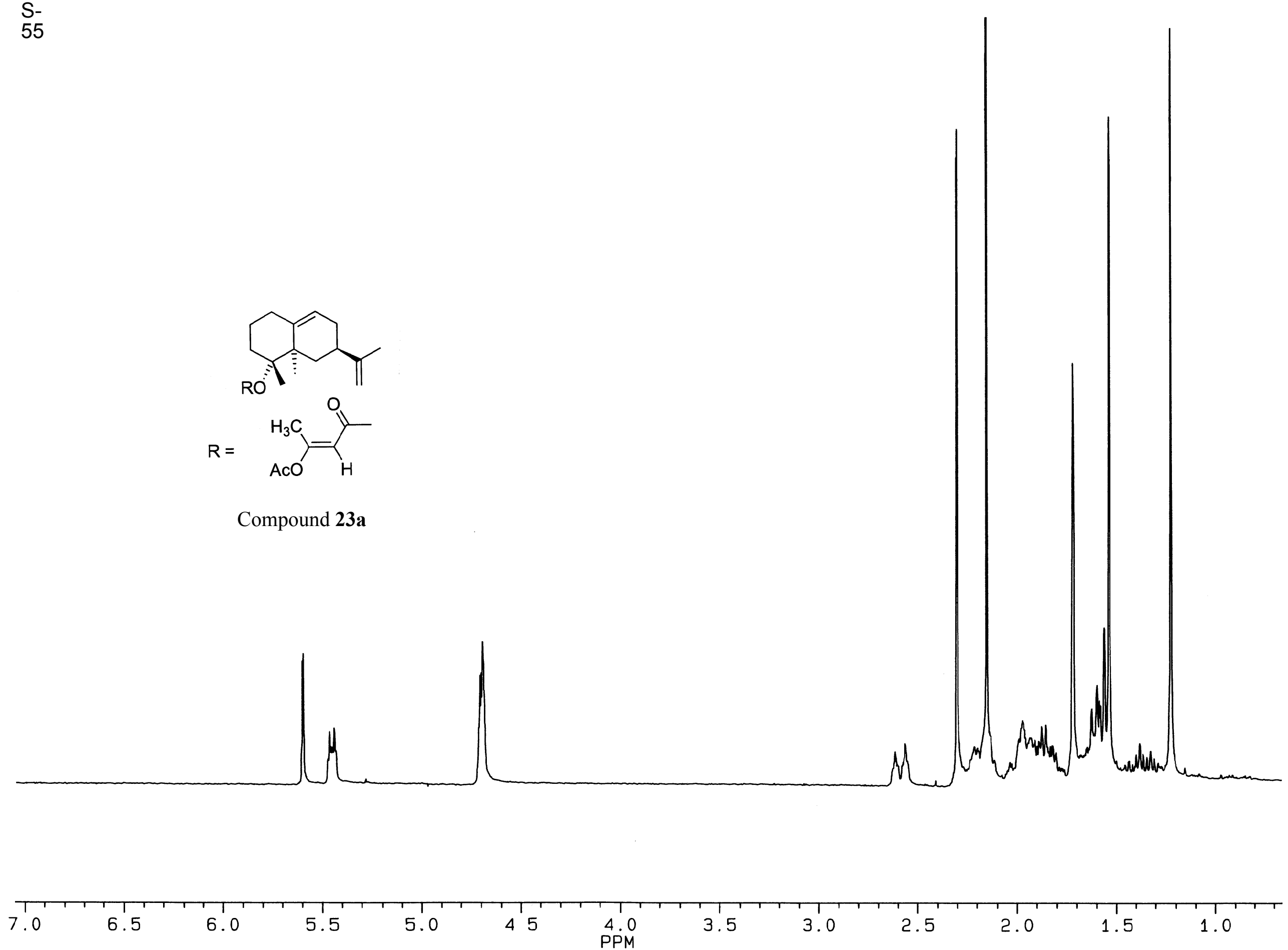
S-
56
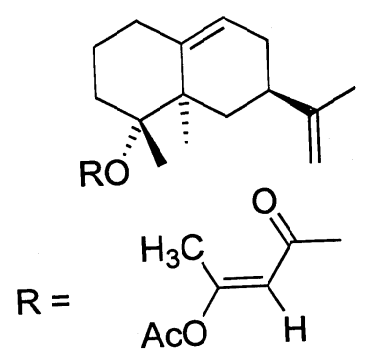

Compound 23a

160.0

140.0

120.0

100.0 80.0

60.0

40.0

20.0 


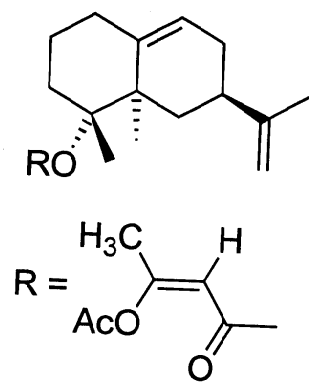

Compound 23b

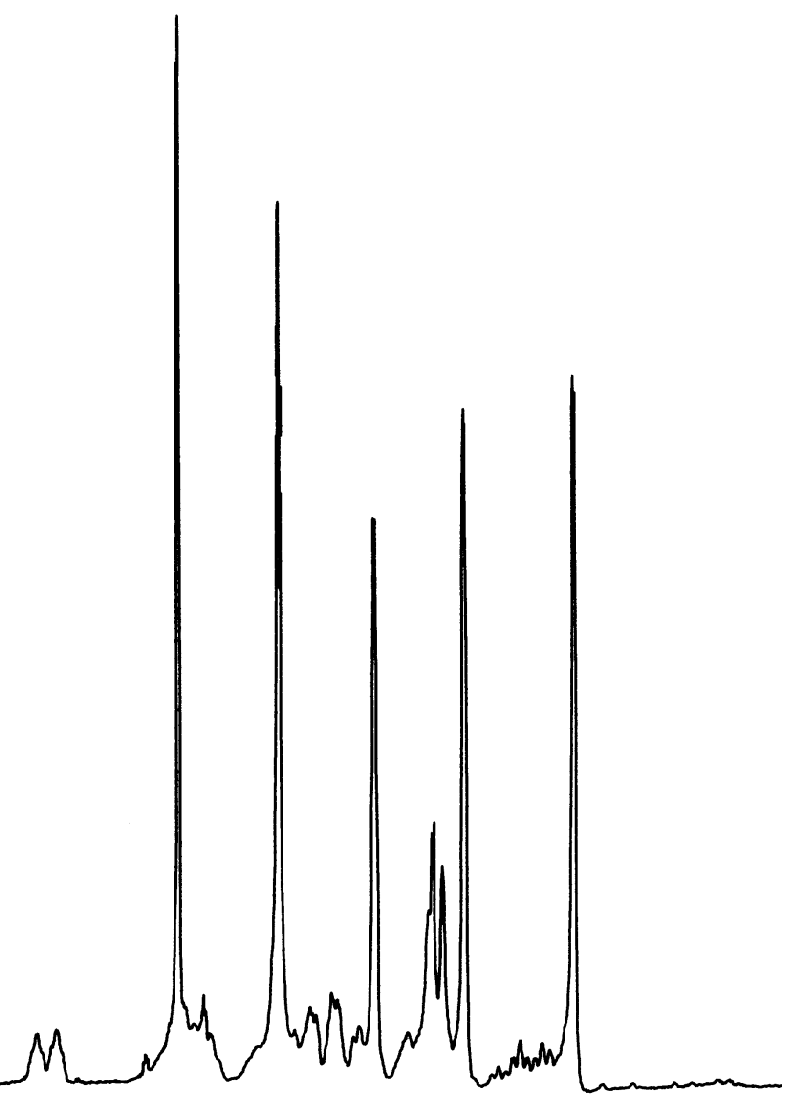




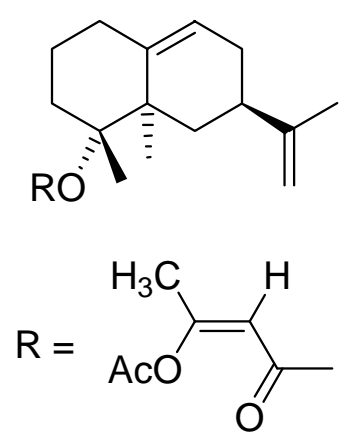

Compound 23b 


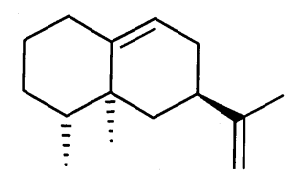

Compound 24

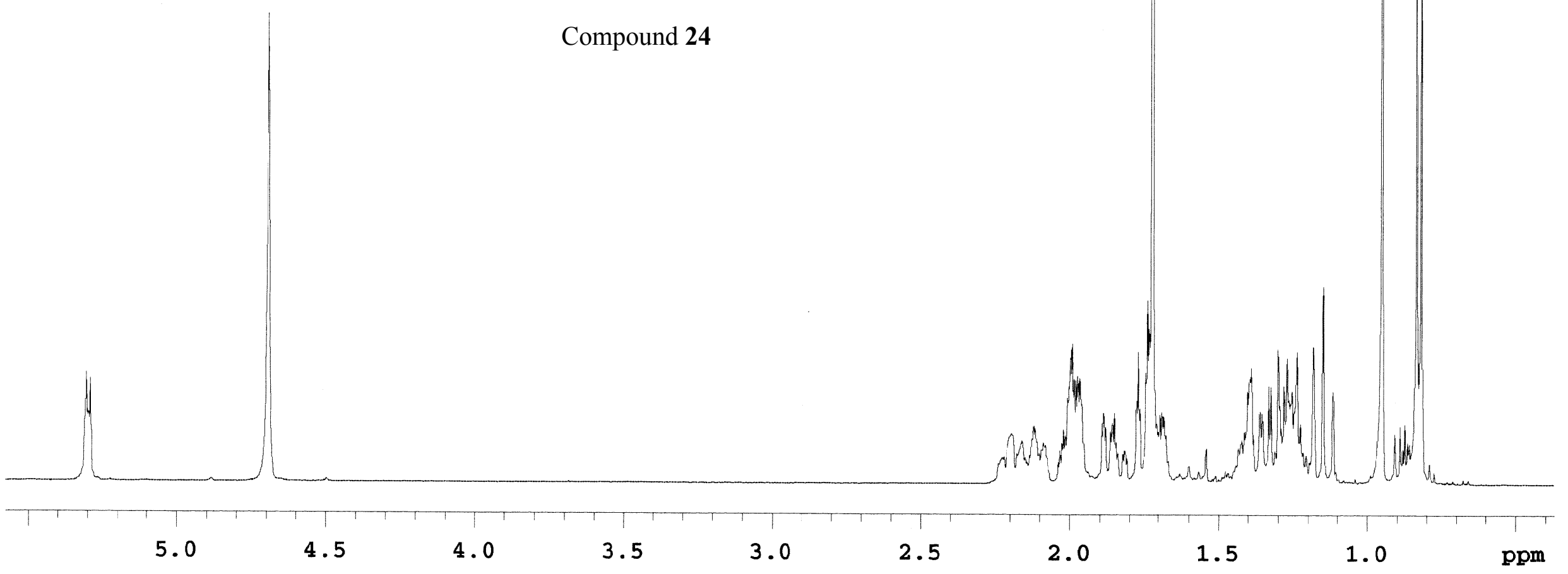


S-

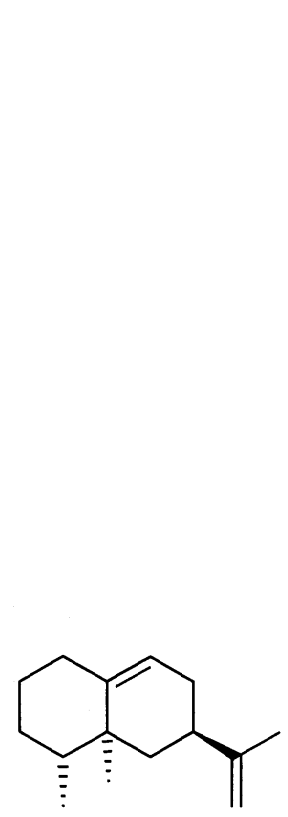

Compound 24

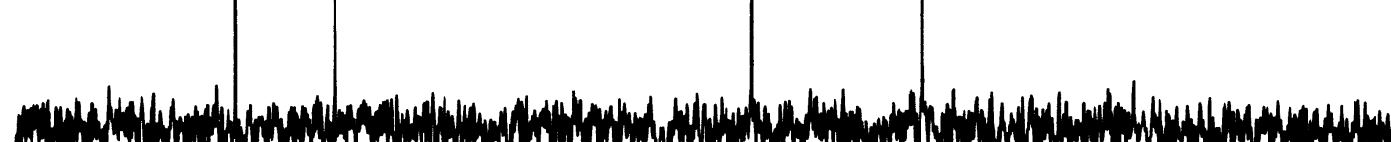

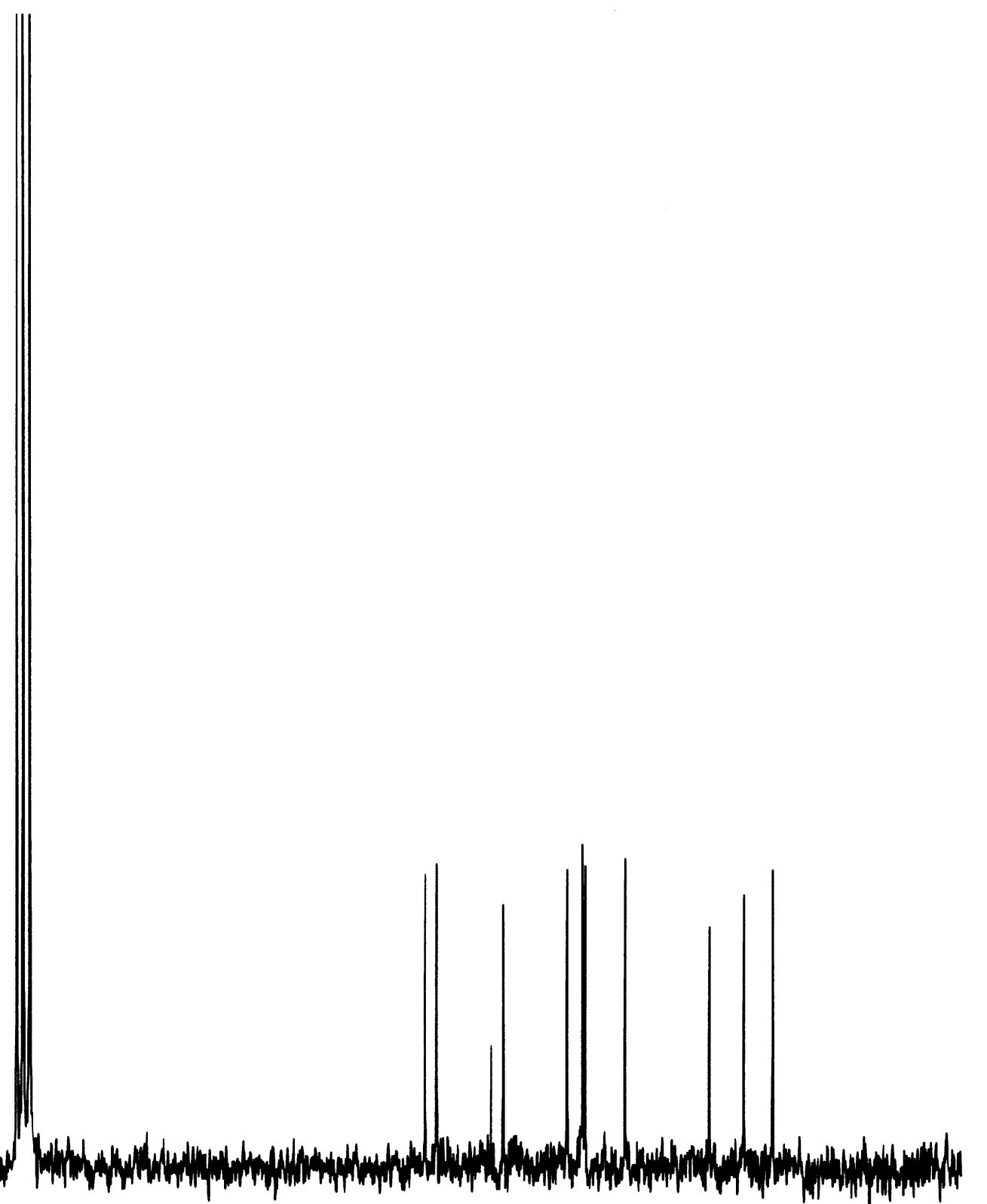

